Revue des patrimoines

13 | 2010

Art rupestre : la 3D un outil de médiation du réel invisible?

\title{
La 3D au service de la conservation des grottes ornées, l'exemple de Lascaux et du simulateur Lascaux
}

Delphine Lacanette et Philippe Malaurent

\section{(2) OpenEdition}

\section{Journals}

Édition électronique

URL : http://journals.openedition.org/insitu/6793

DOI : 10.4000/insitu.6793

ISSN : 1630-7305

\section{Éditeur}

Ministère de la culture

Référence électronique

Delphine Lacanette et Philippe Malaurent, « La 3D au service de la conservation des grottes ornées, l'exemple de Lascaux et du simulateur Lascaux », In Situ [En ligne], 13 | 2010, mis en ligne le 16 avril 2012, consulté le 19 avril 2019. URL : http://journals.openedition.org/insitu/6793 ; DOI : 10.4000/ insitu.6793

Ce document a été généré automatiquement le 19 avril 2019

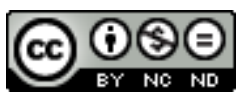

In Situ Revues des patrimoines est mis à disposition selon les termes de la licence Creative Commons Attribution - Pas d'Utilisation Commerciale - Pas de Modification 4.0 International. 


\title{
La 3D au service de la conservation des grottes ornées, l'exemple de Lascaux et du simulateur Lascaux
}

\author{
Delphine Lacanette et Philippe Malaurent
}

\section{Introduction}

1 La grotte de Lascaux, découverte en 1940 et située en Dordogne, est inscrite au Patrimoine mondial de l'Unesco. Depuis sa découverte, plusieurs problèmes sont apparus, à cause du grand nombre de visites ${ }^{1}$ et le dégagement de vapeur d'eau et de dioxyde de carbone qui a causé la formation de calcite et l'apparition d'algues vertes et de mousses. Le ministre de la culture André Malraux a fait fermer la grotte en 1963. Dès lors, des préhistoriens, archéologues, géologues, hydrogéologues, biologistes ont travaillé ensemble pour maintenir la grotte dans l'état le plus stable possible, en utilisant des centrales de mesure pour enregistrer les variations de température, d'hygrométrie, de dioxyde de carbone et développer des traitements et des processus complexes pour éliminer les micro-organismes ${ }^{2}{ }^{3}$.

2 La grotte de Lascaux fait partie du réseau supérieur fossile d'un karst en grande partie comblé par un remplissage argilo-sableux. Ses dimensions sont modestes, la longueur totale est de $250 \mathrm{~m}$, la hauteur maximale des galeries est de $12 \mathrm{~m}$. Ces dernières sont à $24 \mathrm{~m}$ de profondeur au maximum. Son volume accessible est inférieur à $3000 \mathrm{~m}^{3}$. La grotte de Lascaux est considérée comme une cavité superficielle.

Depuis les travaux de la première commission scientifique de 1963, il a été établi que les convections se tiennent dans la cavité naturellement une partie de l'année tandis qu'elles sont maintenues artificiellement le reste de l'année. Pour que cette convection se réalise, la cavité étant en pente, la température de l'air dans les parties profondes doit être plus élevée que la température de l'air près de la surface. Celle des parties profondes varie peu au cours du temps et constitue le résultat d'un équilibre fin entre le transfert de chaleur 
par le sol, les variations de température à la surface, les calories portées par les fluides qui s'infiltrent (eau, vapeur, dioxyde de carbone) et les transferts de chaleur aux parois (changement de phase, convection, radiation).

Plusieurs phénomènes ont été observés sur les parois calcaires et sont néfastes pour la conservation: la corrosion (attaque de la calcite utilisée comme support des peintures préhistoriques menant à la disparition totale des œuvres) et la précipitation de la calcite recouvrant les peintures. La paroi est le lieu privilégié des échanges de chaleur et d'humidité entre le support et l'atmosphère souterraine. La simulation est utilisée pour comprendre ces phénomènes complexes et leurs conséquences sur la conservation de la cavité.

\section{Figure 1}

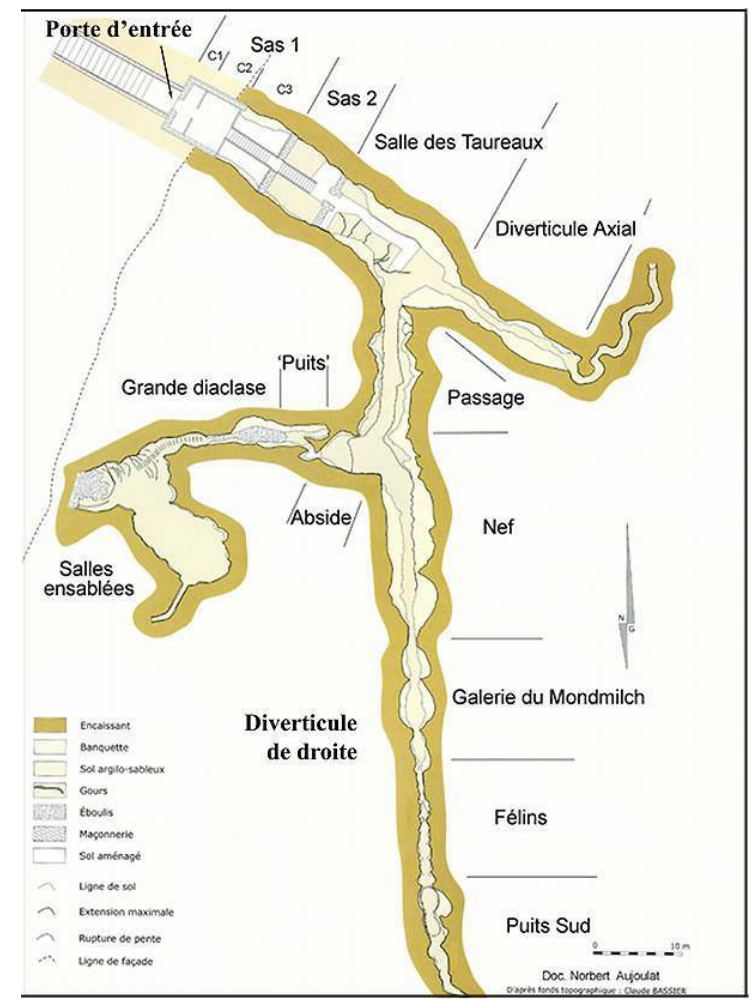

Lascaux. Plan de la cavité, d'après N. Aujoulat sur un fond topographique de Claude Bassier de 1966 Aujoulat, Norbert. LAscauX : Le geste, L'EsPACE ET LE TEMPS. Paris : Seuil, 2004. Norbert Aujoulat (c MCC-CNP

Le simulateur Lascaux est basé sur un code de mécanique des fluides numérique adapté à cet environnement particulier. Cet outil numérique est conçu pour aider le comité scientifique à prendre les décisions concernant les installations et les interventions dans la grotte grâce à l'aspect prédictif de la simulation. Les relations étroites entre le suivi climatique, mené depuis 1963, et le simulateur permet la validation des résultats de la simulation et la proposition de nouveaux modèles de compréhension des phénomènes impliqués dans la caractérisation des conditions favorables à la conservation des peintures et des gravures de la grotte. 


\section{Rappel des faits depuis 2000 dans Lascaux}

6 Suite à l'état des lieux réalisé par Jean-Michel Geneste, conservateur de la grotte, en mai 1995, et soulignant la vétusté du dispositif d'assistance climatique, le changement à l'identique du matériel est préconisé. Des travaux importants sont ainsi réalisés dans la Salle des Machines, à la suite desquels apparaissent des moisissures blanches en 2001. Cependant, bien avant cette crise, des études émettaient déjà des doutes sur la stabilisation et l'invulnérabilité de la grotte, et confirmaient ce qui avait été remarqué vingt-cinq ans plus tôt, suite à l'épisode de 1963, la très faible marge de manœuvre pour assurer des conditions optimales de conservation ${ }^{4}$.

\section{Point sur la situation climatique}

7 La compréhension des mécanismes responsables de l'équilibre dynamique de la grotte est complexe. La grotte est dans une situation naturelle évolutive ; le suivi de cette évolution a mis en évidence dès 1963 que six mois par an, l'air souterrain peut condenser sur les parois décorées; le processus d'évaporation est possible les six autres mois. Ces mécanismes sont déclenchés par la modification des températures en surface de la roche comme dans l'air souterrain et peuvent provoquer des phases de corrosion ou de dépôt de calcite en liaison avec la présence de gaz carbonique.

8 Le but recherché est de restreindre les possibilités de condensation en diminuant les écarts entre les valeurs de la pression partielle de vapeur d'eau de l'air et, celles calculées, de la pression partielle saturante, correspondant à la température de la roche, afin de réduire les risques de condensation sur celle-ci. L'utilisation des échanges aérodynamiques, qui se font naturellement par convection entre la partie axiale (salle des Taureaux et diverticule axial) et la salle des Machines, a permis la mise au point d'un système de régulation de l'atmosphère. Il limite la possibilité de condensation de la vapeur d'eau sur les parois décorées; la condensation est favorisée artificiellement dans la salle des Machines. Concrètement, cette circulation (naturelle ou artificielle suivant les époques de l'année) assure le refroidissement de l'air et sa perte d'un excès d'humidité (au contact d'échangeurs thermiques). Selon la période, cette perte d'humidité se fait également au contact de la roche et de la toiture de la salle des Machines qui recueille les eaux de la nappe aquifère temporaire située au-dessus de la cavité. Dans ces conditions, la perturbation climatique saisonnière détectée par H. Schoeller et P.M. Guyon pouvait être maîtrisée.

\section{Inversion naturelle des températures à l'intérieur de Lascaux}

9 La figure 2 présente les courbes de température dans le sol à différentes profondeurs (10, 15 et $20 \mathrm{~m}$ ) calculées grâce à une loi de conduction monodimensionnelle (Fourier). La figure 3 correspond aux températures dans la roche mesurées en deux points caractéristiques de la cavité, le fond du diverticule axial (DFR) à une profondeur de $20 \mathrm{~m}$ et la voûte de la salle des Taureaux (TVR) à une profondeur de $10 \mathrm{~m}$. L'examen de ces courbes permet de distinguer différentes périodes. De 1965 à 1981, les conditions thermiques souterraines liées à la propagation des ondes thermiques externes sont favorables à l'établissement d'un régime de circulation de l'air par convection entre le 
fond du diverticule axial et la salle des Taureaux. Depuis 1981, ce régime de circulation de l'air par convection est affecté par l'impact de l'évolution climatique extérieure et se traduit par l'inversion des températures dans la cavité (inversion des courbes). De 1970 à 1981, les températures mesurées dans la roche au fond du diverticule axial sont supérieures à celles mesurées à la voûte de la salle des Taureaux, tandis que depuis 1981 on mesure l'inverse. Cette inversion est responsable des conditions de moins en moins favorables à l'établissement de convections vers la salle des Machines. Elle affecte la régulation du climat souterrain telle qu'elle avait été définie par les chercheurs de la commission d'études scientifiques en 1963. (fig. $\mathrm{n}^{\circ} 2, \mathrm{n}^{\circ}$ 3)

Figure 2

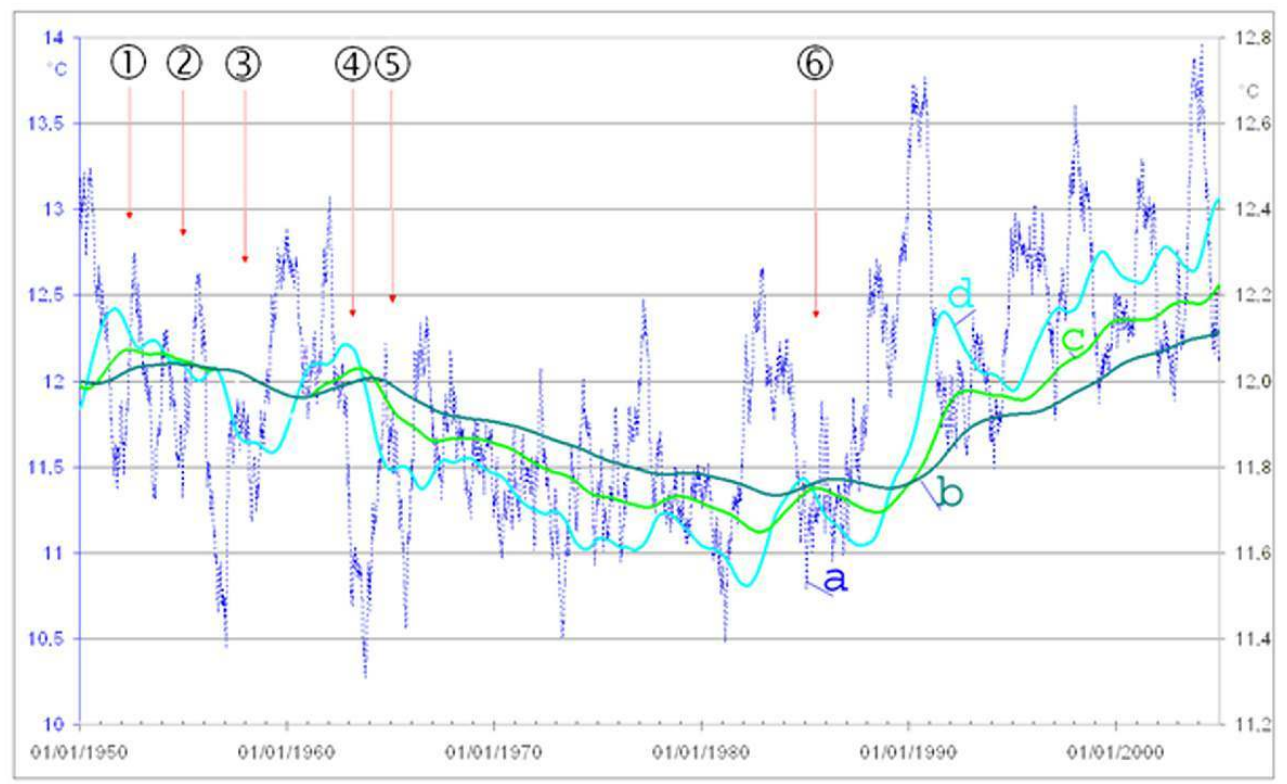

Évolution de la température mesurée en surface (axe des ordonnées entre 10 et $14^{\circ} \mathrm{C}$ ) à la station météorologique de Gourdon (courbe a) et évolution théorique calculée de la température (axe des ordonnées entre 11,2 et $12,8^{\circ} \mathrm{C}$ ) dans le sol à différentes profondeurs (courbe b à $20 \mathrm{~m}$ sous le sol, courbe c à $15 \mathrm{~m}$ sous le sol, courbe d à $10 \mathrm{~m}$ sous le sol) entre les années 1950 et 2004

Doc. D. Lacanette, P. Malaurent. (c) D. Lacanette, P. Malaurent 
Figure 3

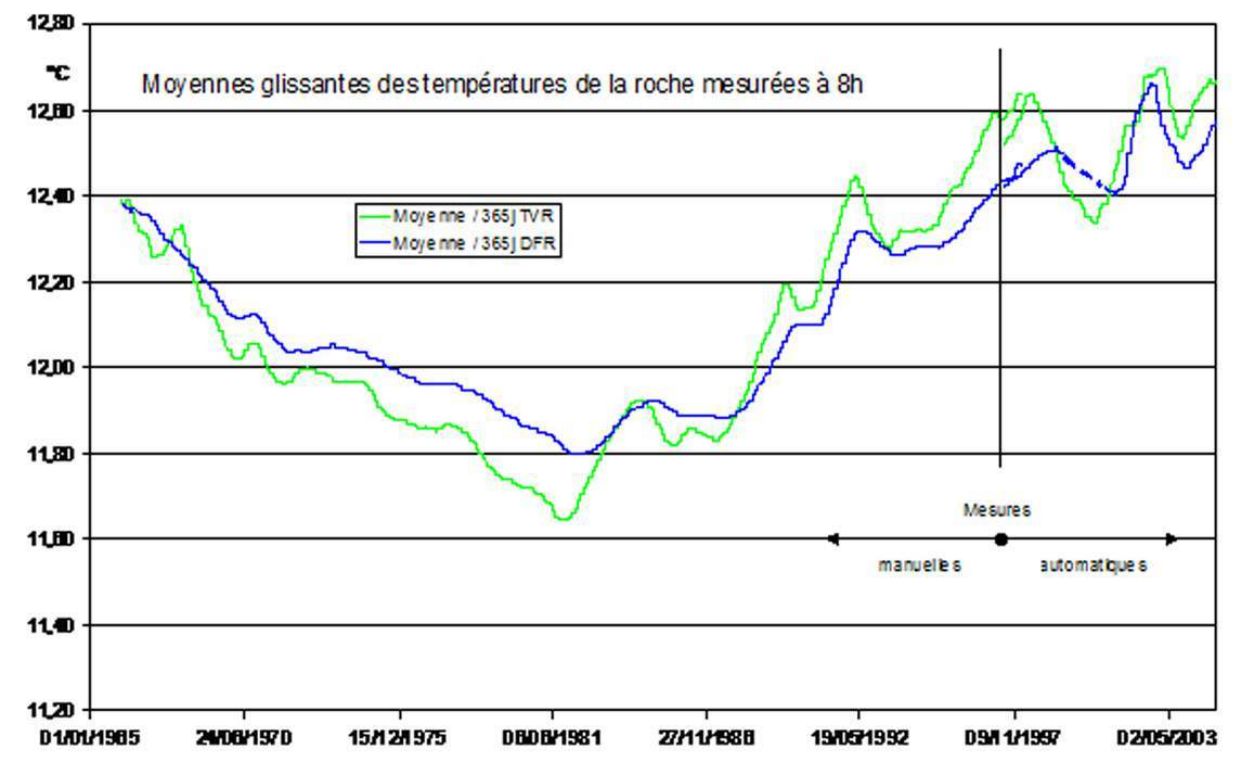

Évolution mesurée de la température dans la cavité, à différentes profondeurs sous le sol : voûte de la Salle des Taureaux (TVR) et fond du Diverticule axial (DFR)

Doc. D. Lacanette, P. Malaurent. ( ) D. Lacanette, P. Malaurent

La figure 4 présente l'évolution théorique de la température en fonction de la profondeur à différentes périodes. De 1965 à 1981, le gradient de température dans la cavité est conforme au schéma de fonctionnement défini par la commission de 1963. En hiver, l'ordre des températures est croissant de la surface vers la profondeur ; en été, cet ordre est maintenu artificiellement par abaissement de la température de l'air de la salle des Machines. L'inertie thermique du sol augmente au fur et à mesure que l'épaisseur de sol concernée augmente. Ainsi depuis 1981, les températures des parties profondes deviennent plus faibles que la température moyenne de surface. Le gradient naturel des températures s'est inversé. Ce phénomène est indépendant du système de régulation artificielle de la cavité. Il est à relier exclusivement au régime climatique extérieur. (fig. $\mathbf{n}$ $\circ 4)$ 


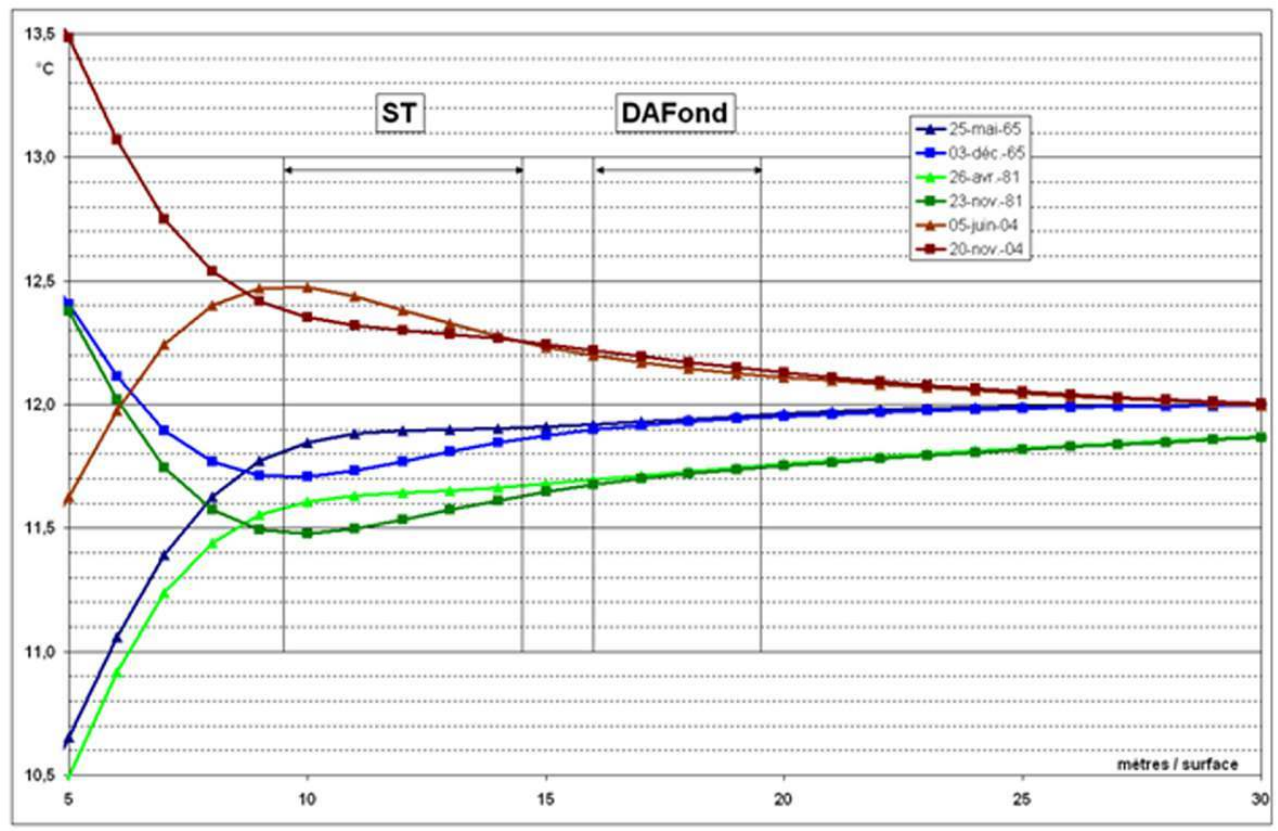

Évolution théorique de la température en fonction de la profondeur, suivant les années (1965-1981-2004) à différentes dates

Doc. D. Lacanette, P. Malaurent. @ D. Lacanette, P. Malaurent

\section{Le simulateur Lascaux : un outil d'aide à la décision}

Le simulateur Lascaux est basé sur un code de mécanique des fluides et tient compte de la géométrie complexe de la grotte grâce à une approche des domaines fictifs ${ }^{5}{ }^{6}$. L'intérêt principal de la modélisation réside dans son aspect prédictif. Il est possible d'évaluer les conséquences à court terme des modifications des installations dans la cavité ou les modifications dans le système d'assistance climatique. Les premières simulations par des modèles numériques ont rendu possible la vérification des premières hypothèses proposées par la première commission scientifique. Les différentes étapes de ce travail ont permis la mise au point d'une méthodologie d'aide à la conservation. En réalisant la simulation de scénarios ayant déjà eu lieu, les hypothèses proposées par l'observation ou par l'analyse de données sont validées ou invalidées numériquement.

\section{Objectifs}

12 L'instrumentation de la grotte est très importante, cependant, les limites sont parfois atteintes en terme de sensibilité des appareils de mesure. Il est à rappeler que les conditions thermiques et hydriques dans la cavité sont complexes; l'humidité est supérieure à $98 \%$ et la température est comprise entre $11^{\circ} \mathrm{C}$ et $13^{\circ} \mathrm{C}$ suivant les années, avec des variations annuelles de l'ordre du dixième de degré.

13 La simulation est largement utilisée dans de nombreux domaines, comme l'industrie aéronautique ou sidérurgique, pour atteindre des échelles d'espace et de temps qui ne peuvent pas être atteintes par d'autres méthodes. La simulation numérique en mécanique 
des fluides est appliquée aujourd'hui à la conservation de la grotte de Lascaux. La simulation requiert l'expérimentation et l'instrumentation pour valider les modèles, dans le but de prédire les écoulements thermoaérauliques, et de fournir des informations sur des scénarios divers. En ce sens, cet outil a été créé pour répondre aux questions du Comité scientifique international de la grotte de Lascaux et le travail est réalisé en synergie avec les autres composantes du comité.

Le simulateur Lascaux est utilisé pour la réalisation de scénarios, comme l'influence des aménagements (échafaudages, cloisons, machinerie), l'influence du climat (introduction de conditions instationnaires) et l'influence de la présence humaine (amélioration de la répartition du temps de présence).

15 Finalement, il a été conçu pour pouvoir être facilement adapté à d'autres grottes ornées. Les modèles sont communs à tous les milieux souterrains, la géométrie étant le seul paramètre à modifier dans le simulateur.

\section{Le maillage est la base du simulateur}

16 La première étape de la simulation est la génération du maillage qui constitue la base du simulateur. Un relevé 3D a été réalisé par le cabinet de géomètre Perazio par laser. Il est visualisé à la figure 5 à l'aide du logiciel d'images de synthèse 3D Studio Max. Le relevé 3D est triangularisé sur sa surface comme cela peut être visualisé à la figure 6, chaque triangle est composé de 3 nœuds. Ensuite, chaque nœud est projeté sur un maillage cubique de la grille structurée cartésienne, et le nouveau maillage est montré sur la même vue que précédemment à la figure 7. Finalement, la projection dans notre code de calcul, Thétis (Aquilon) développé au laboratoire TREFLE, est présentée figure 8. (fig. $\mathbf{n}^{\circ} \mathbf{5}, \mathbf{n}^{\circ} \mathbf{6}$, $\left.\mathrm{n}^{\circ} 7, \mathbf{n}^{\circ} 8\right)$ 
Figure 5

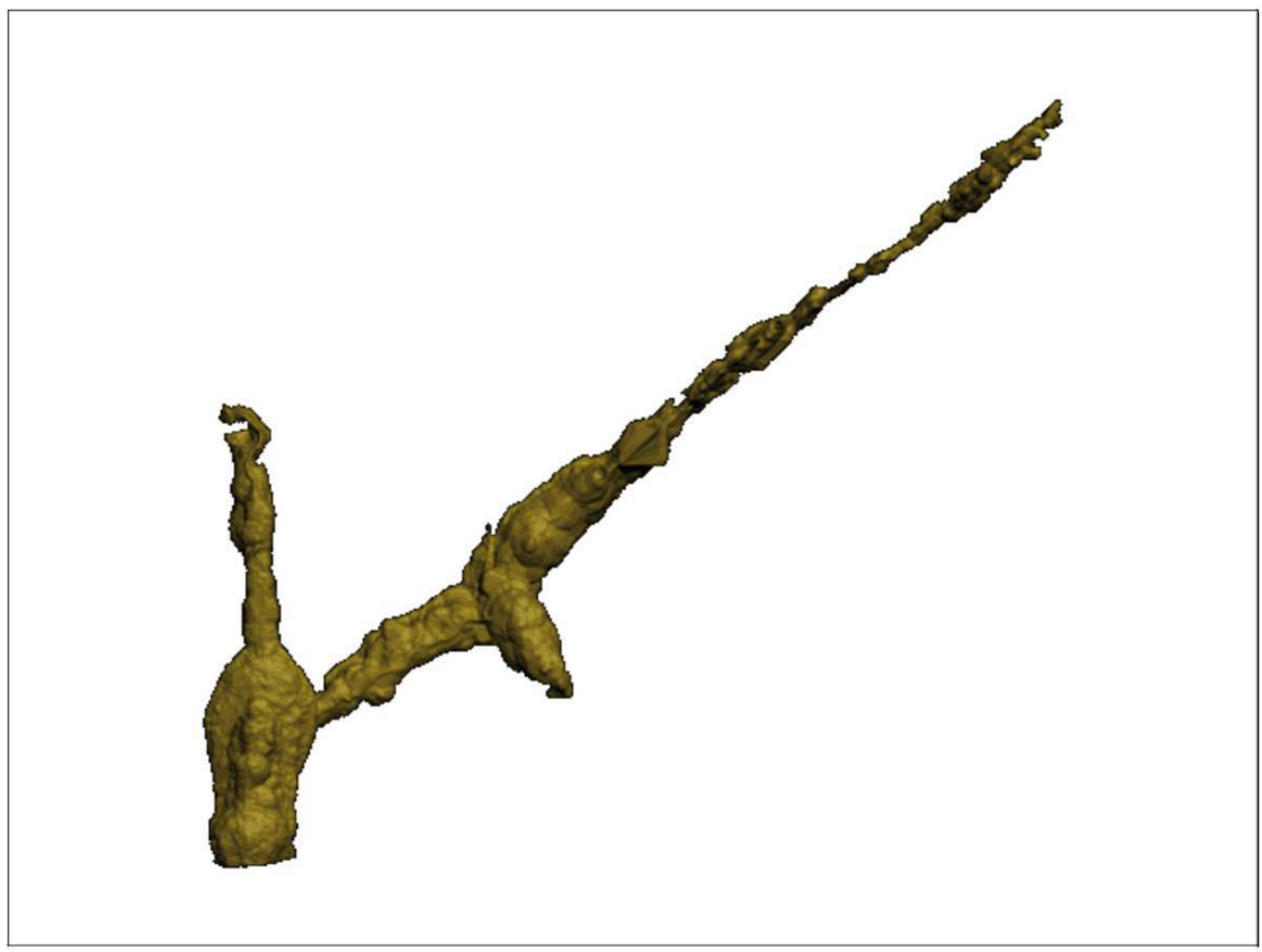

Relevé laser 3D fourni par le cabinet de géomètre Perazio

Doc. Cabinet Perazio. (c) Ministère de la Culture et de la Communication, Cabinet Perazio

Figure 6

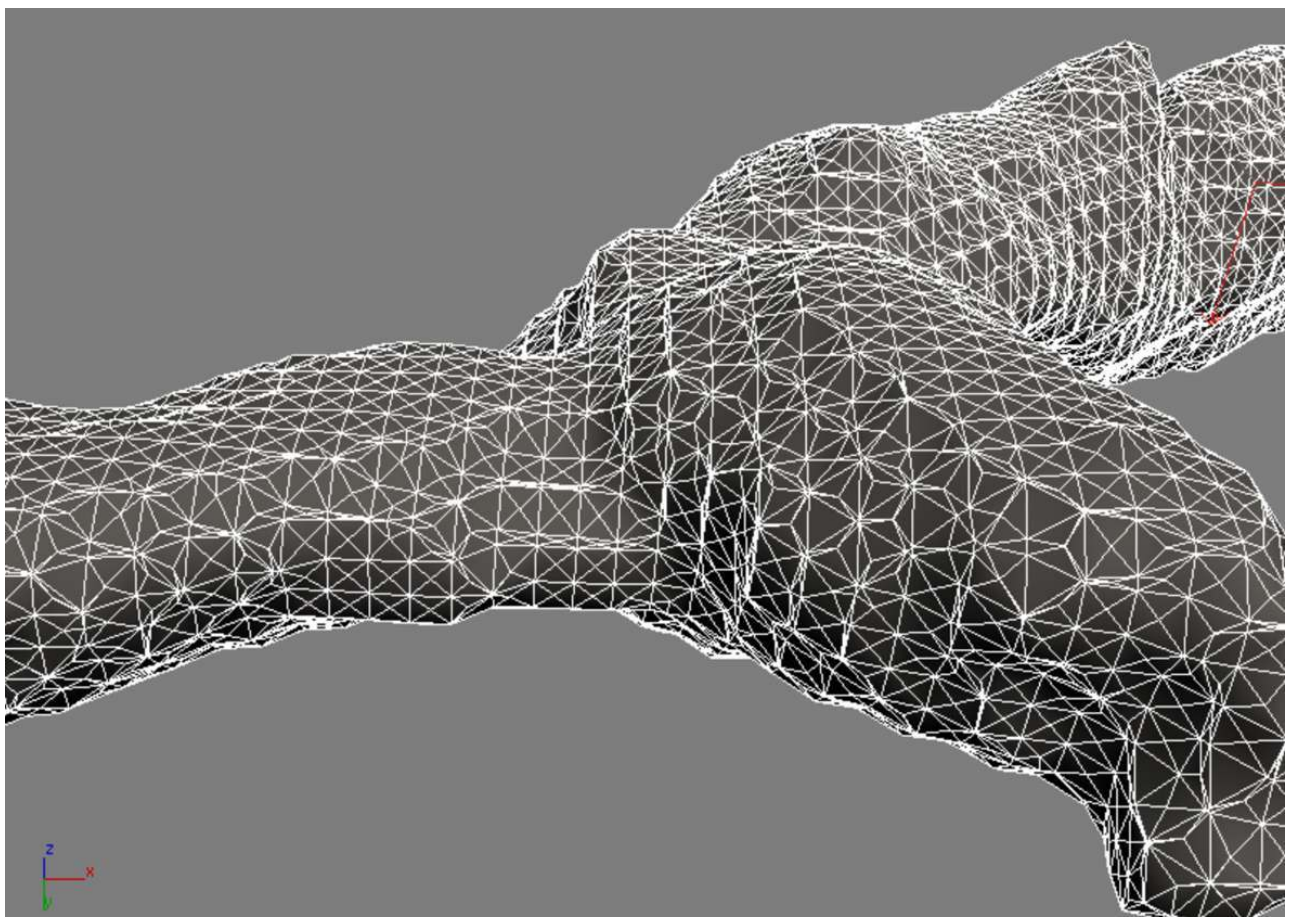

Détail du relevé 3D

Doc. Cabinet Perazio. ( ) Ministère de la Culture et de la Communication, Cabinet Perazio 
Figure 7

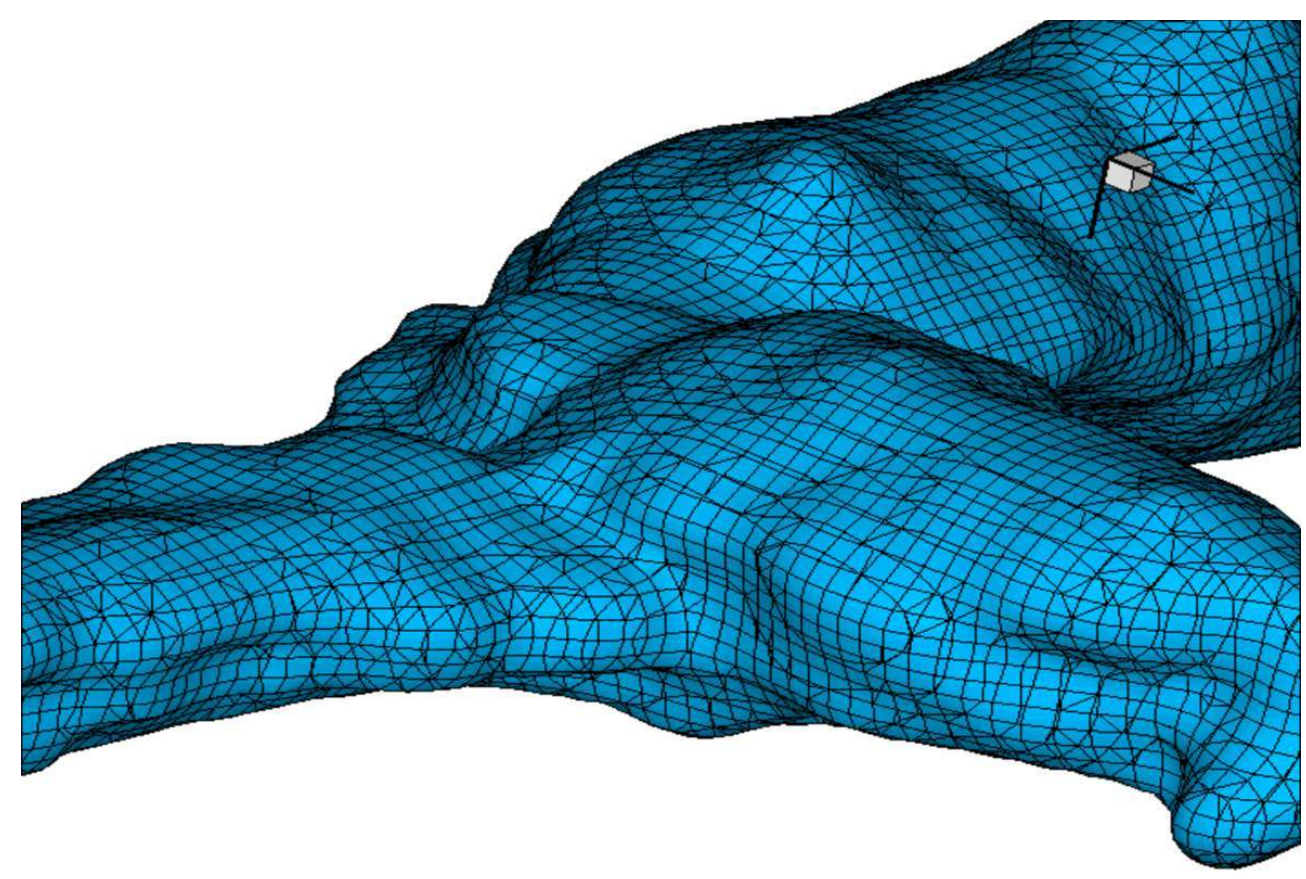

Détail de la projection dans Aquilon

(C) Laboratoire TREFLE 


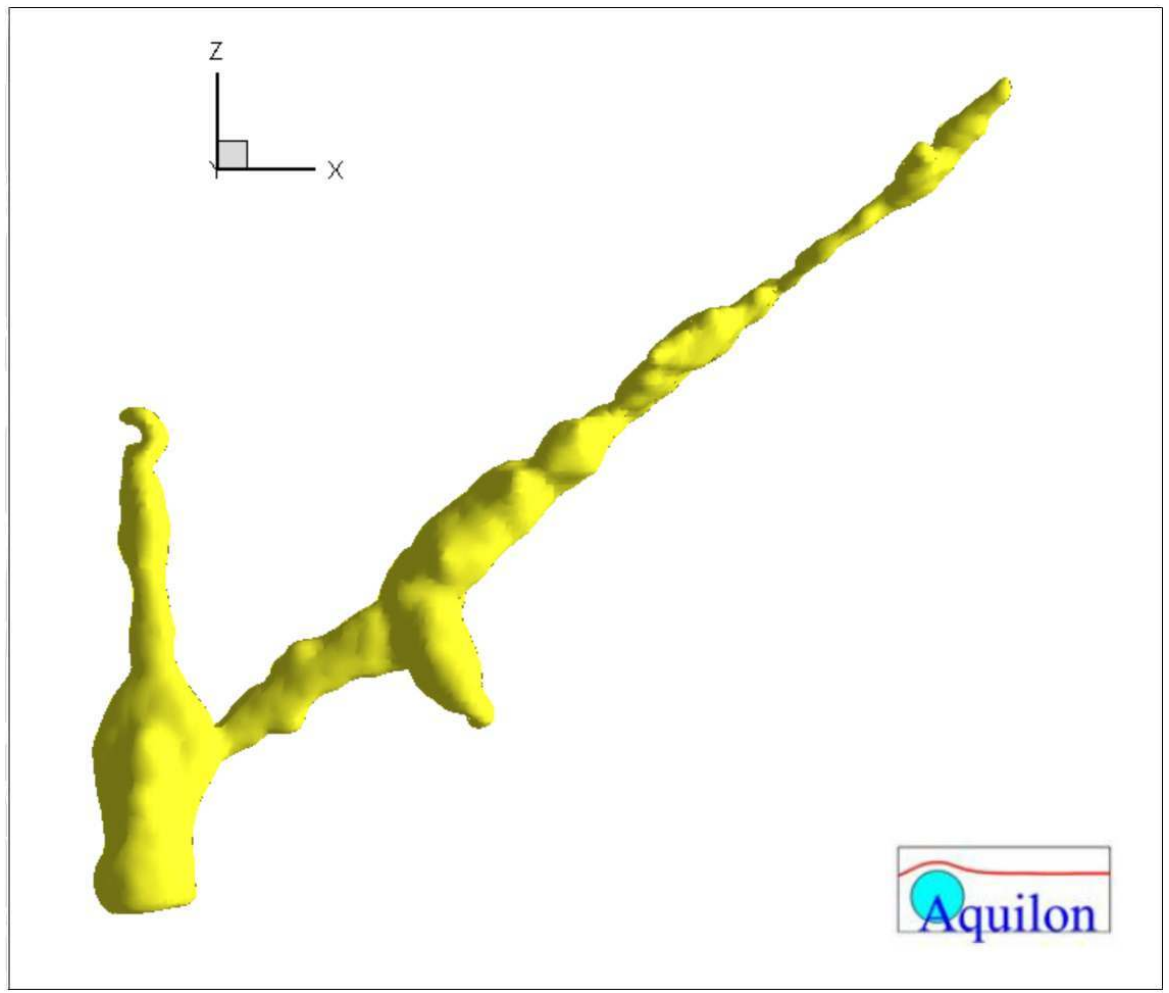

Projection dans Aquilon

(c) Laboratoire TREFLE

17 Sur chaque nœud de la grille cartésienne composant le maillage sont résolues les équations constitutives de la mécanique des fluides et les paramètres importants (température, vitesse, taux d'humidité) seront fournis sur ces nœuds.

\section{Équations constitutives et modèles}

Dans le milieu fluide, les équations de conservation décrivant les écoulements de convection incompressibles instationnaires d'un fluide newtonien et l'évolution de la concentration de l'humidité, sous l'hypothèse de Boussinesq, sont les équations de Navier-Stokes (1-2), de l'énergie (3) et du transport de la concentration en humidité (4) écrites sous la forme de la vitesse et de la température : 


$$
\nabla \cdot u=0
$$

$$
\begin{gathered}
\rho\left(\frac{\partial u}{\partial t}+(u \cdot \nabla) u\right)=\rho g-\nabla p+\nabla \cdot\left(\mu\left(\nabla u+\nabla^{\prime} u\right)\right) \\
\rho C_{P}\left(\frac{\partial T}{\partial t}+(u \cdot \nabla) T\right)=\nabla \cdot(\lambda \nabla T) \\
\left(\frac{\partial C}{\partial t}+\mathbf{u} \cdot \nabla \phi\right)=\nabla \cdot D \nabla \phi
\end{gathered}
$$

où $u$ est le vecteur vitesse, $\rho$ la densité, $t$ le temps, $g$ le vecteur gravité, $p$ la pression, $\mu$ la viscosité, $C_{\mathrm{P}}$ la capacité calorifique, $\lambda$ la conductivité, $\mathrm{C}$ le taux d'humidité absolue et $\mathrm{D}$ le coefficient de diffusion.

Figure 9

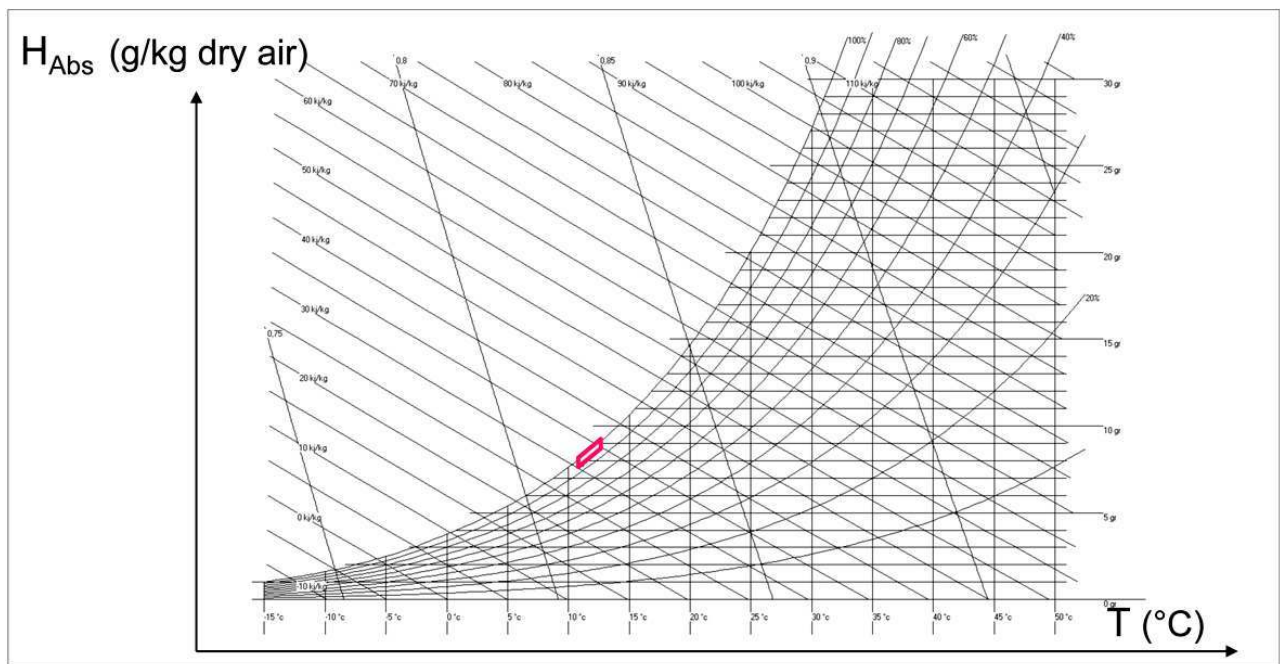

Diagramme psychrométrique

Doc. D. Lacanette, P. Malaurent. @ D D. Lacanette, P. Malaurent

Le terme source $\mathrm{F}_{\mathrm{C}}$ dans l'équation de transport est la variation du taux d'humidité partout à la paroi en fonction du gradient de température.

L'humidité est initialisée à une valeur de 98 \% d'humidité relative dans l'ensemble de la grotte. Les calculs sont faits sur le taux d'humidité absolue, qui est donné par la température en chaque point grâce au diagramme psychrométrique (fig. $\mathbf{n}^{\circ}$ 9). Le diagramme psychrométrique présente graphiquement la relation entre la température de l'air et le taux d'humidité absolue. La région entourée concerne les conditions rencontrées dans la grotte.

\section{Vue de la grotte de Lascaux dans le simulateur}




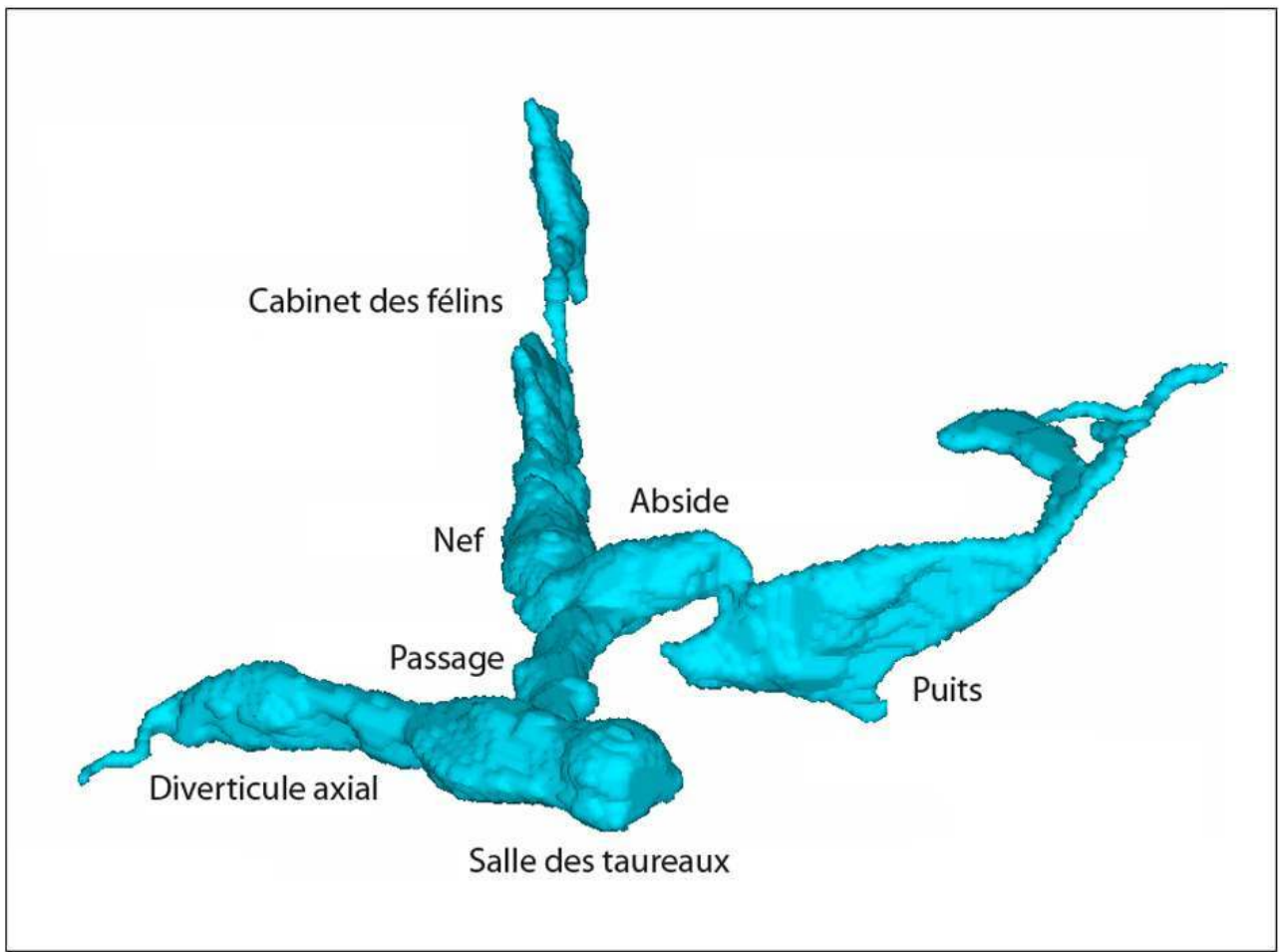

Vue générale de la grotte de Lascaux dans le simulateur

Doc. D. Lacanette, P. Malaurent. (c) D. Lacanette, P. Malaurent

La grotte de Lascaux est présentée à la figure 10 telle qu'elle est prise en compte dans le simulateur. Des repères indiquant les différentes salles ont été placés pour se repérer par rapport au plan, mieux connu. (fig. $\left.\mathbf{n}^{\circ} \mathbf{1 0}\right)$

\section{Conditions limites définies par des mesures}

Des conditions limites doivent être ajoutées au simulateur pour recréer les conditions réelles de température dans la grotte. La figure 11 présente l'évolution de l'amplitude de la température moyenne pour chaque mois de l'année, sous abri, sur trente ans, comme une fonction de la profondeur dans le sol, de la surface de la colline jusqu'à 20 mètres de profondeur. L'évolution de la température est périodique. Sur 20 mètres, l'amplitude décroît pendant une année de $15,3^{\circ} \mathrm{C}$ à $0,025^{\circ} \mathrm{C}$. Comme la grotte de Lascaux est située entre 10 et 20 mètres, l'amplitude maximum de la température est environ de $0,6^{\circ} \mathrm{C}$ pendant l'année. (fig. $\left.\mathbf{n}^{\circ} \mathbf{1 1}\right)$ 
Figure 11
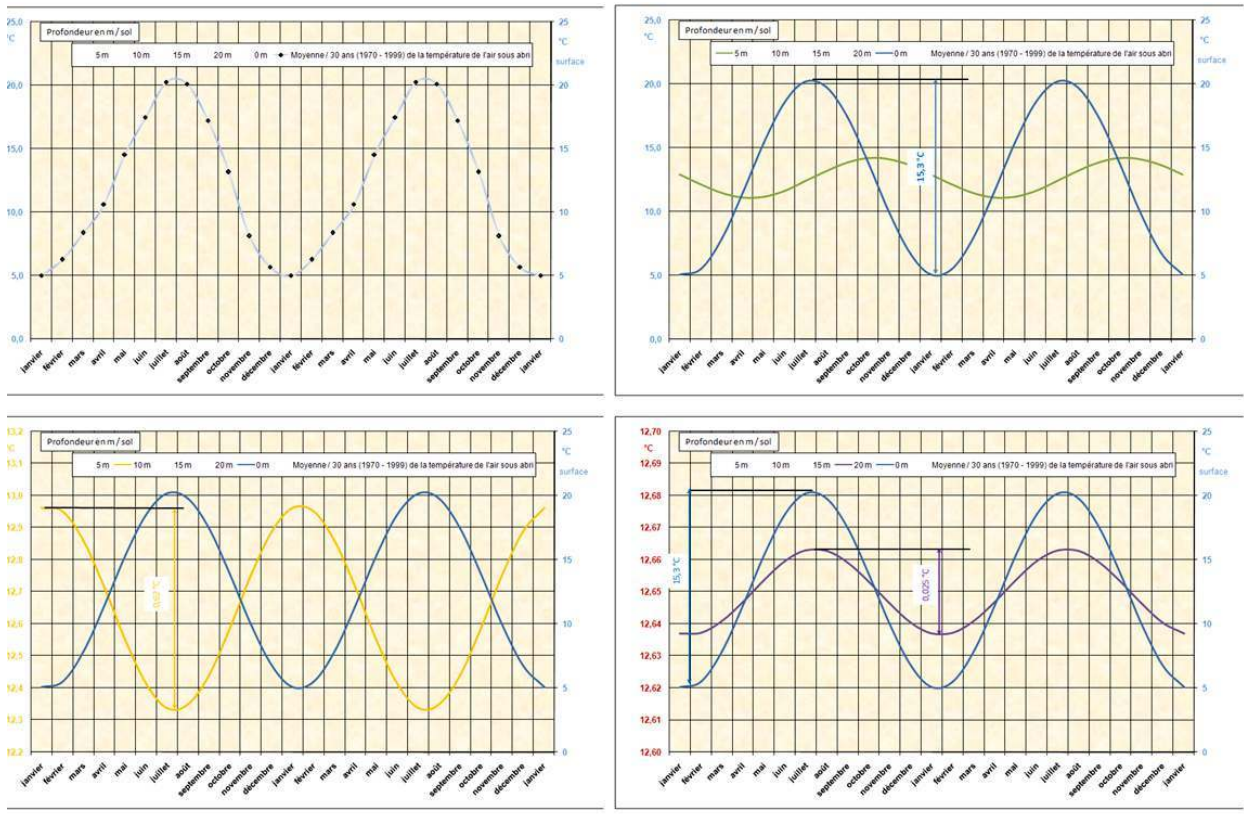

Évolution de l'amplitude de la température en fonction de la profondeur dans le sol de la surface de la colline jusqu'à $20 \mathrm{~m}$ de profondeur (en haut à gauche : surface de la colline, en haut à droite : $5 \mathrm{~m}$ de profondeur, en bas à gauche : $10 \mathrm{~m}$ de profondeur, en bas à droite : $20 \mathrm{~m}$ de profondeur). Attention, les échelles de températures sont différentes d'un graphique à l'autre

Doc. D. Lacanette, P. Malaurent. (c) D. Lacanette, P. Malaurent

La figure 12 présente l'évolution de la différence de phase de la température en fonction de la profondeur. La différence de phase sur les températures aux parois en fonction de la température de surface à 20 mètres de profondeur est de 12 mois. Concernant la grotte de Lascaux, la différence de phase est d'au moins 6 mois. Les températures les plus élevées sont présentes en hiver, quand les températures extérieures sont les plus basses. (fig. $\mathbf{n}$ -12) 

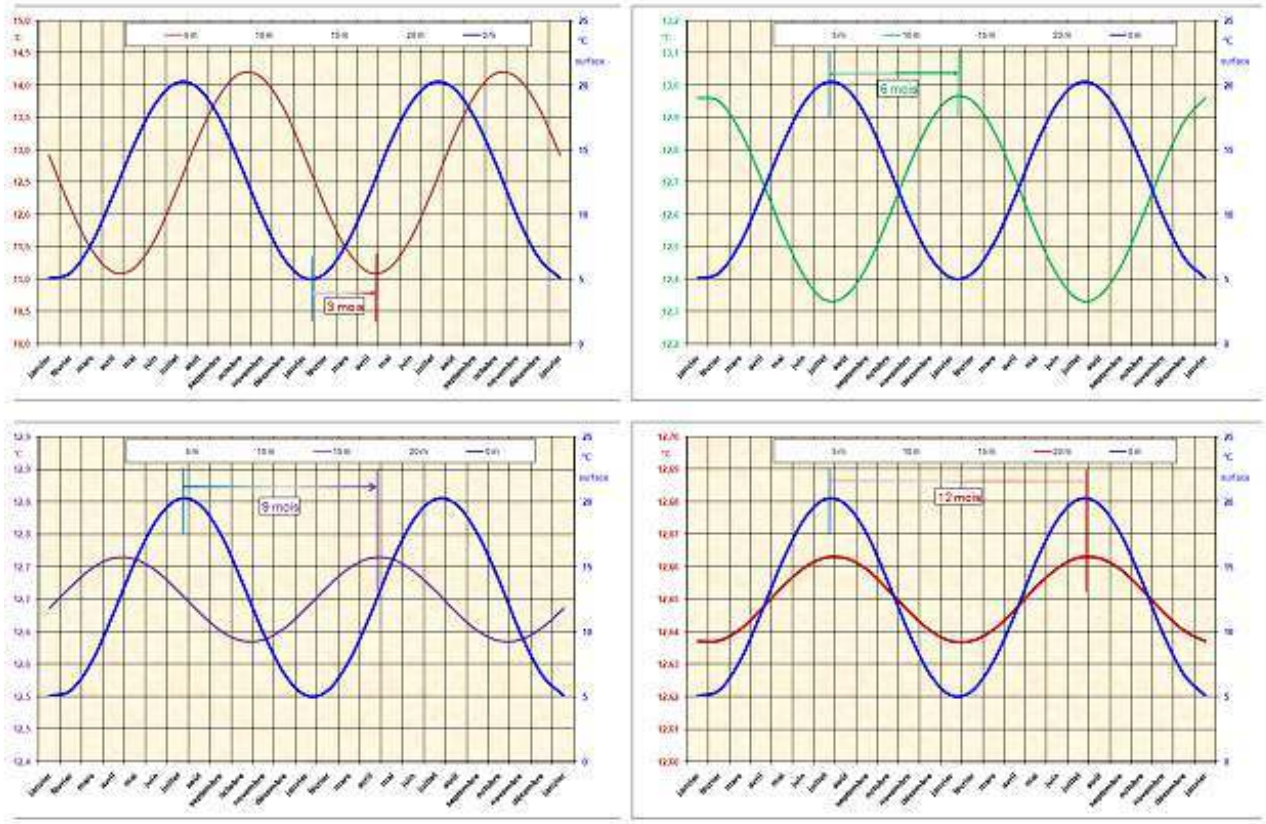

Évolution de la différence de phase de la température en fonction de la profondeur dans le sol de la surface de la colline jusqu'à $20 \mathrm{~m}$ de profondeur (en haut à gauche : $5 \mathrm{~m}$ de profondeur en haut à droite : $10 \mathrm{~m}$ de profondeur, en bas à gauche : $15 \mathrm{~m}$ de profondeur, en bas à droite : $20 \mathrm{~m}$ de profondeur). Attention, les échelles de températures sont différentes d'un graphique à l'autre Doc. D. Lacanette, P. Malaurent. (c) D. Lacanette, P. Malaurent

Dans le but de prendre en compte aussi précisément que possible les conditions limites aux parois de la grotte, nous considérons la colline environnante et nous initialisons un gradient de température issu de mesures prises à l'intérieur de la cavité. Ainsi, chaque point de la paroi de la grotte sera associé à la valeur de la température correspondant à sa profondeur.

Le profil de température en fonction de la profondeur introduit dans le simulateur a été calculé grâce à un modèle théorique (loi de Fourier) à partir de températures mesurées à la surface de la colline au-dessus de la grotte et présenté à la figure 13. (fig. $\left.\mathbf{n}^{\circ} \mathbf{1 3}\right)$ 


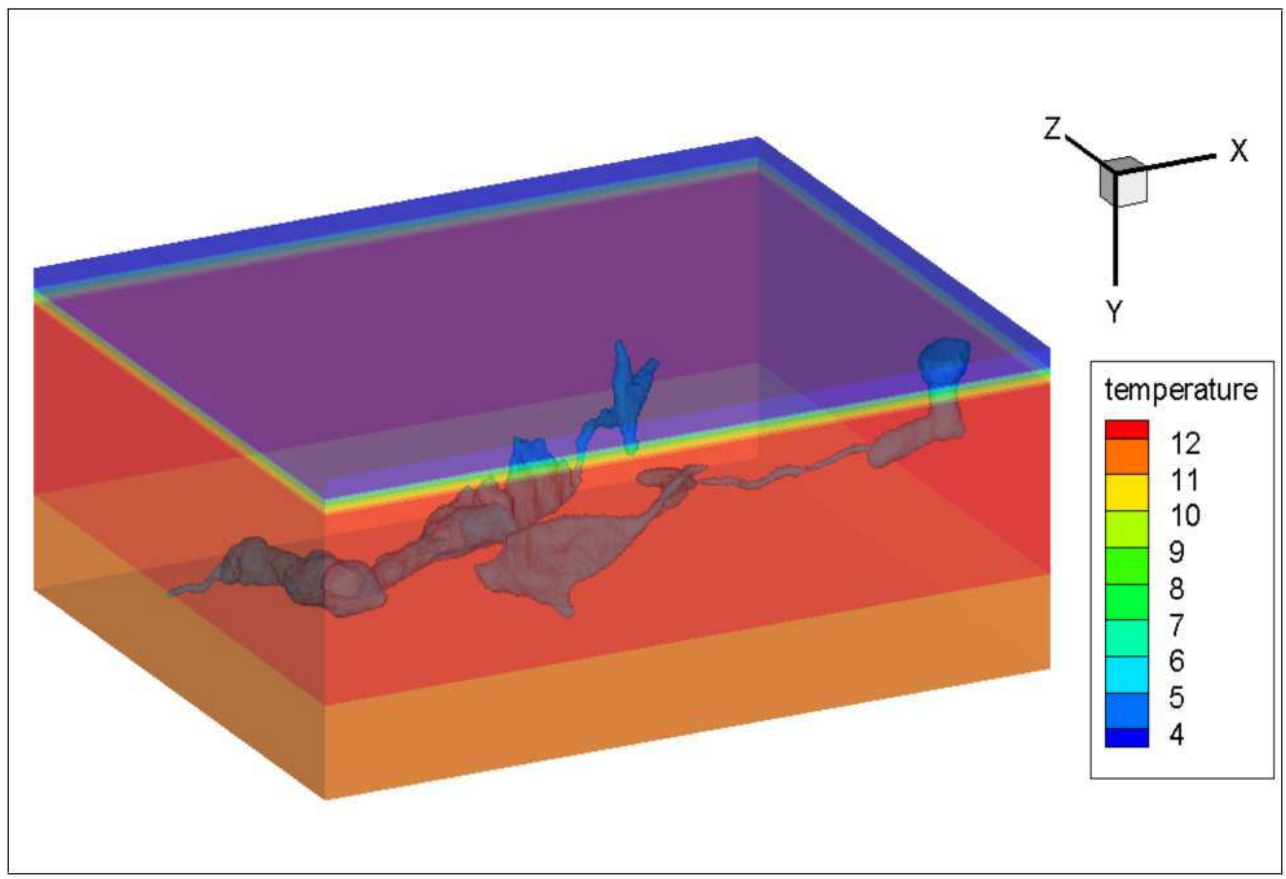

Profil de température en fonction de la profondeur dans la colline entourant la grotte de Lascaux Doc. D. Lacanette, P. Malaurent. (c) D. Lacanette, P. Malaurent

\section{Cas d'étude basés sur les observations}

Les configurations thermiques ont été choisies et leurs conditions initiales et limites correspondantes introduites dans le simulateur. À partir des mesures réalisées dans la cavité depuis cinquante ans, nous avons choisi des cas d'étude qui sont typiques de périodes auxquelles la grotte a eu des comportements caractéristiques.

Dans la grotte, le taux d'humidité relative est proche de $100 \%$. Dans chaque configuration, la zone de condensation est la zone la plus froide, dans laquelle l'air condense. La position de la zone de condensation se déplace le long de la grotte durant l'année, elle est située au point le plus froid de la courbe de température. 


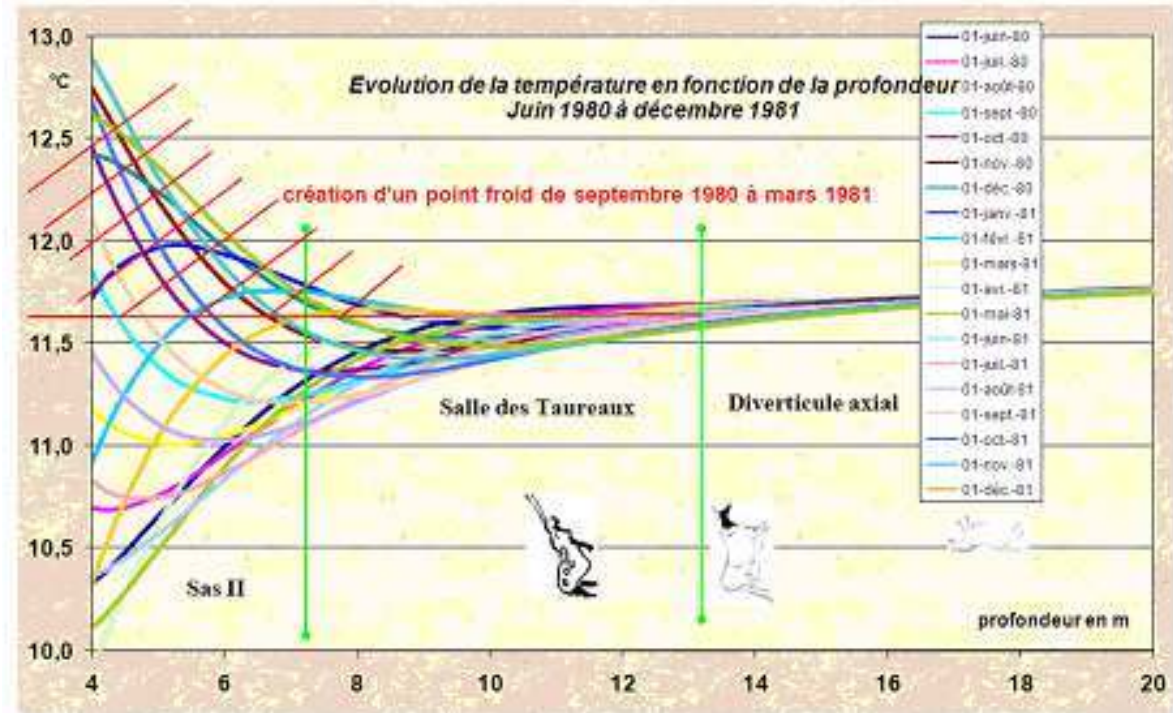

Évolution de la température dans le sol en fonction de la profondeur au début des années 1980 Doc. D. Lacanette, P. Malaurent. ( ) D. Lacanette, P. Malaurent

La configuration thermique de septembre 1981 est étudiée à la figure 14. Elle donne l'évolution de la température dans le sol en fonction de la profondeur de juin 1980 à décembre 1981. Quand la zone de condensation atteint la salle des Taureaux, de septembre 1980 à mars 1981, ceci constitue un problème de conservation. Sur cette figure, on voit que la création d'un point froid dans la salle des Machines de septembre à mars permet le mouvement de la zone de condensation vers le sas II, en abaissant la température dans la zone moins profonde, le sas II. (fig. $\mathbf{n}^{\circ} \mathbf{1 4}$ )

Figure 15

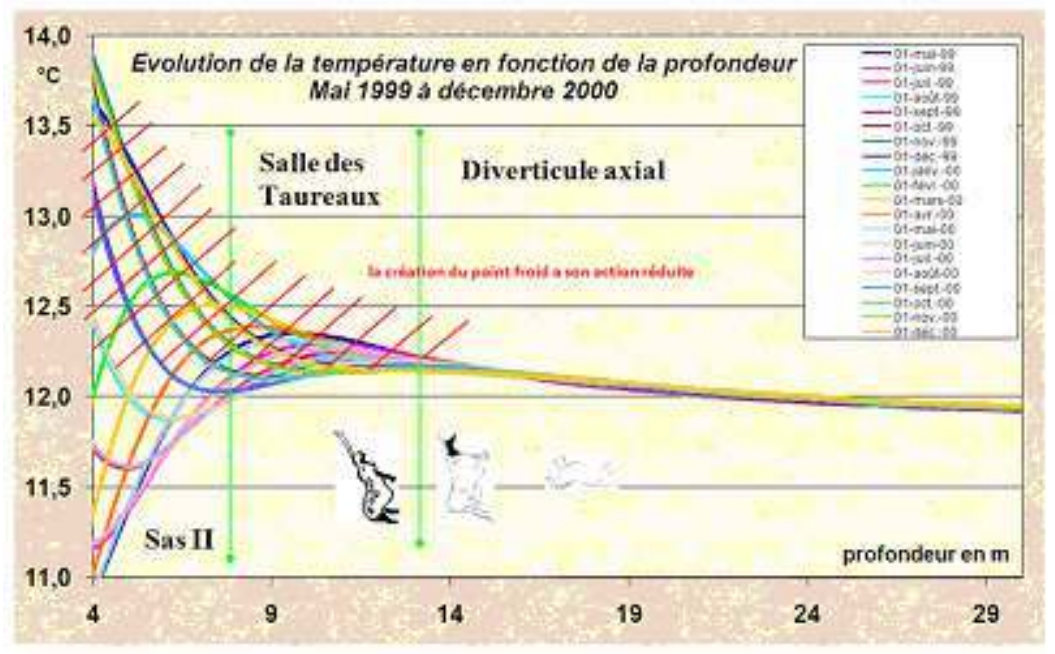

Évolution de la température dans le sol en fonction de la profondeur à la fin des années 1990

Doc. D. Lacanette, P. Malaurent. ( ) D. Lacanette, P. Malaurent

La configuration thermique de décembre 1999 est étudiée à la figure 15. Dans ce cas, nous faisons face à un problème de conservation dans la zone ornée (diverticule axial) à cause 
de l'inversion de la pente de température, entraînant une position différente des zones de condensation par rapport à la configuration thermique de 1981. La figure présente l'évolution de la température dans le sol en fonction de la profondeur de mai 1999 à décembre 2000. La condensation dans la salle des Taureaux est limitée, il n'y a pas d'action de la machinerie dans le diverticule axial, ou cela entraînerait un séchage de l'air trop important dans la salle des Taureaux. (fig. $\left.\mathbf{n}^{\circ} \mathbf{1 5}\right)$

Figure 16

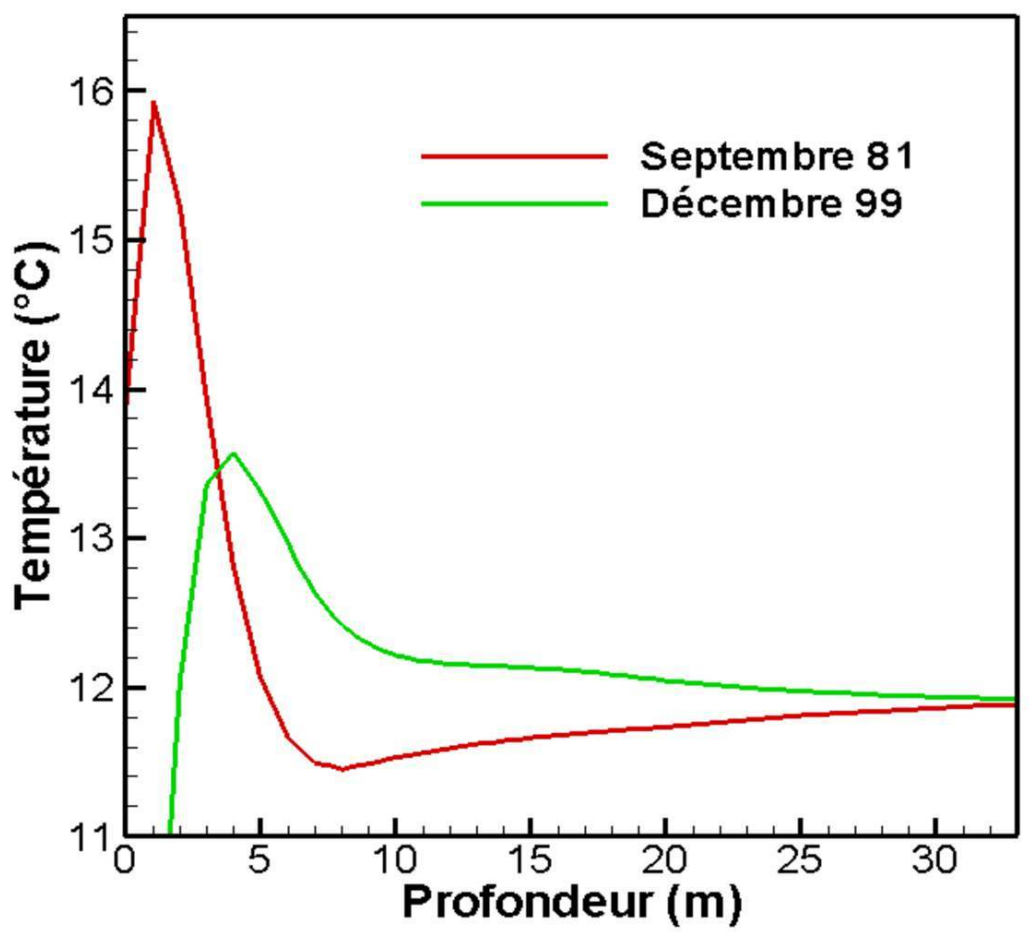

Températures en fonction de la profondeur pour les deux configurations thermiques étudiées Doc. D. Lacanette, P. Malaurent. (c) D. Lacanette, P. Malaurent

31 Se basant sur des mesures et l'analyse vue auparavant, nous avons choisi deux configurations thermiques correspondant à deux périodes très différentes. La première est celle de septembre 1981 ; à cette date le comportement de la cavité est apparemment stable. La seconde est celle de décembre 1999, avant les travaux de remplacement du système de régulation de l'air.

La figure 16 représente les températures en fonction de la profondeur suivant un modèle de conduction de la chaleur dans la roche environnante. La grotte est située entre 10 et 20 mètres de profondeur, dans une zone où la pente est positive en septembre 1981 et négative en décembre 1999. (fig. $\mathbf{n}^{\circ}$ 16)

\section{Résultats de la simulation}

\section{Validation sur des observations}

Un courant d'air est créé par convection naturelle à cause des différences de température entre les voûtes et le sol à l'intérieur de la cavité. Il est matérialisé dans les figures 17, 18, 
19 et 20 par des flèches. En septembre 1981, dans la galerie de droite de la grotte, l'air se dirige naturellement depuis les endroits les plus profonds (galerie du Mondmilch) vers les moins profonds (passage) et vers la salle des Taureaux, tandis qu'en décembre 1999 il vient de la salle des Taureaux et va du passage vers la galerie du Mondmilch. (fig. $\mathbf{n}^{\circ} \mathbf{1 7}, \mathbf{n}$ $\circ 18, n^{\circ} 19, n^{\circ} 20$ )

Figure 17

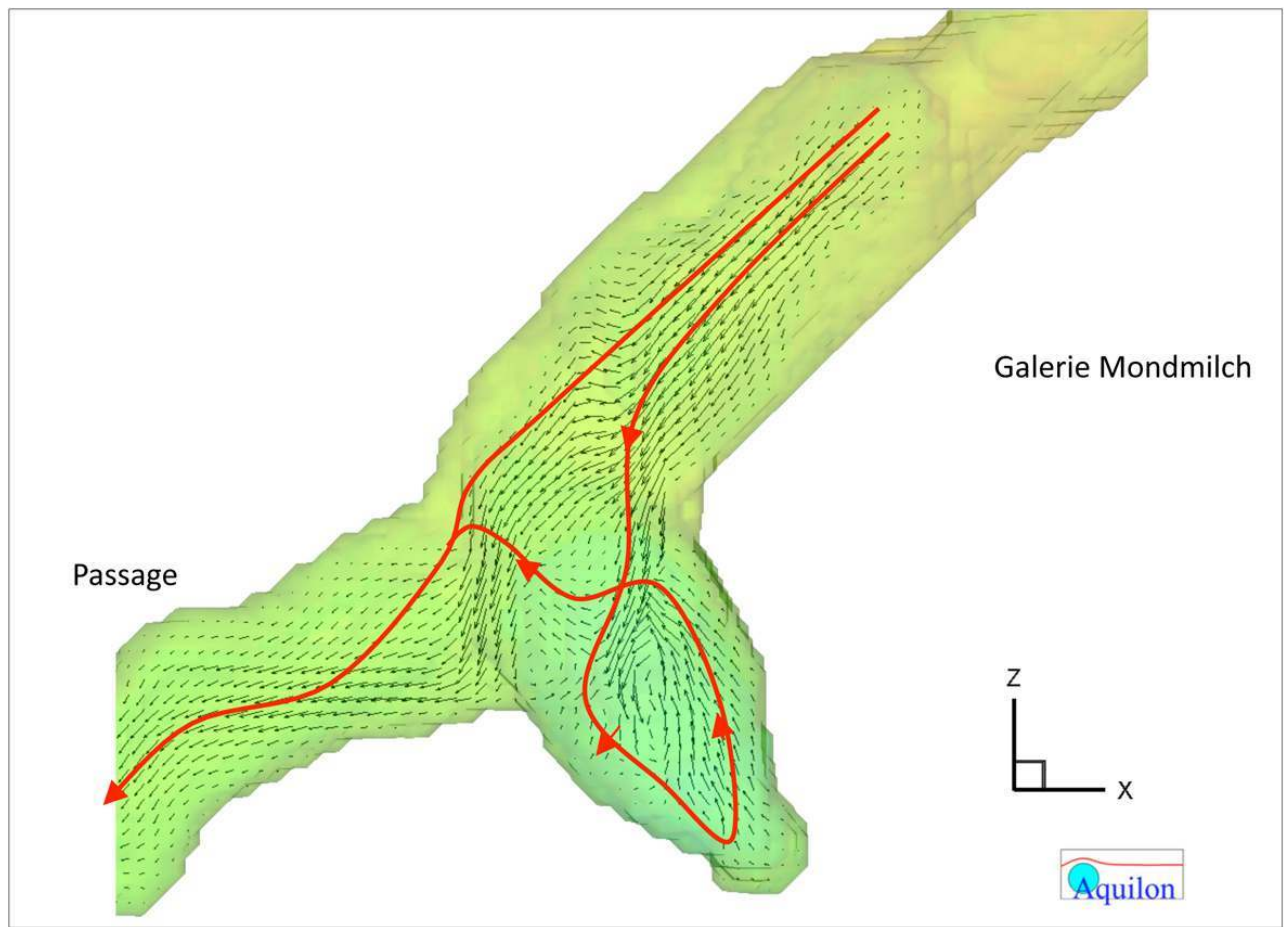

Sens des écoulements dans la galerie de droite dans la configuration climatique de septembre 1981 Doc. D. Lacanette, P. Malaurent. (c) D. Lacanette, P. Malaurent 
Figure 18

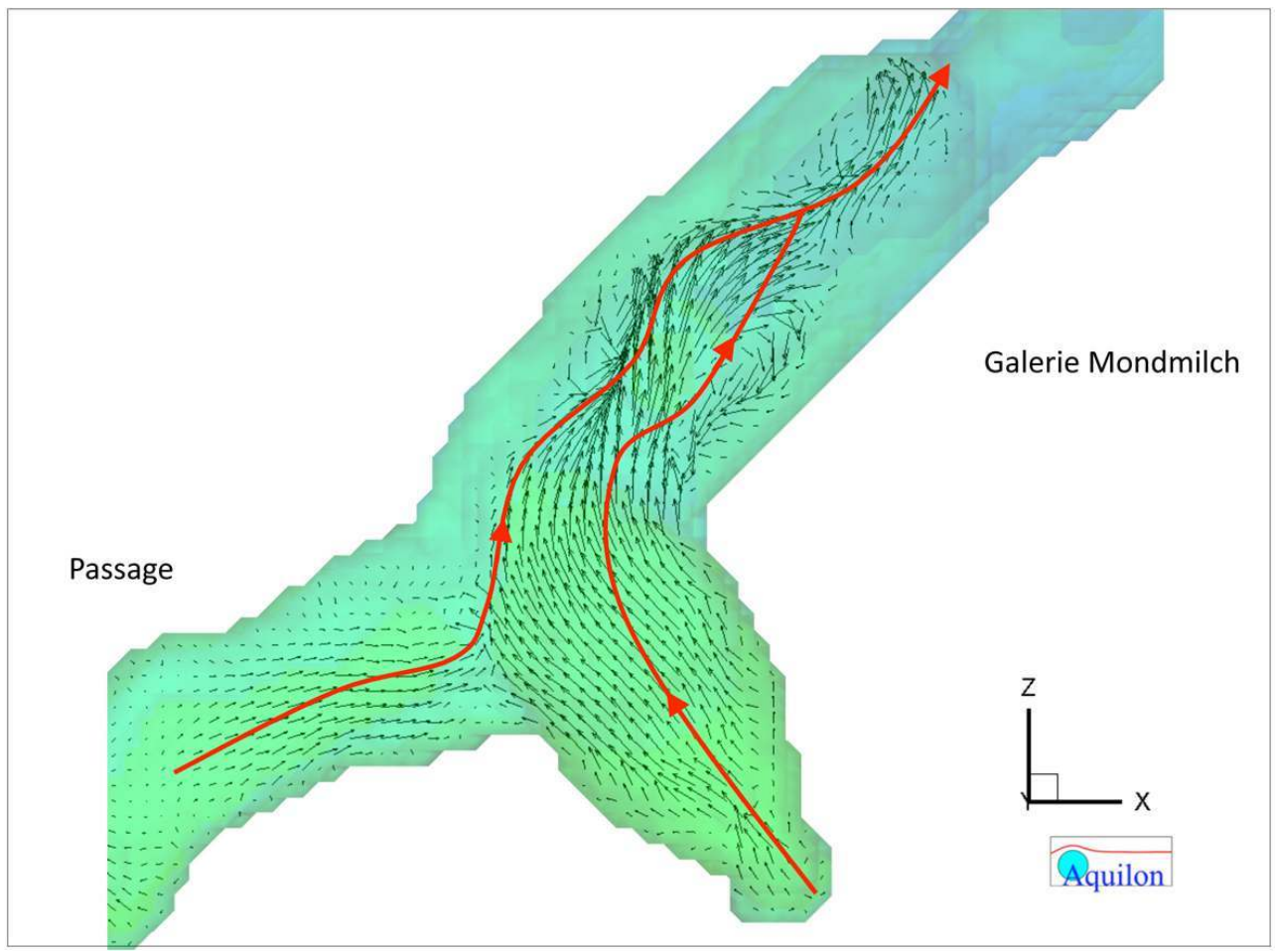

Sens des écoulements dans la galerie de droite dans la configuration climatique de décembre 1999 Doc. D. Lacanette, P. Malaurent. (c) D. Lacanette, P. Malaurent

Figure 19

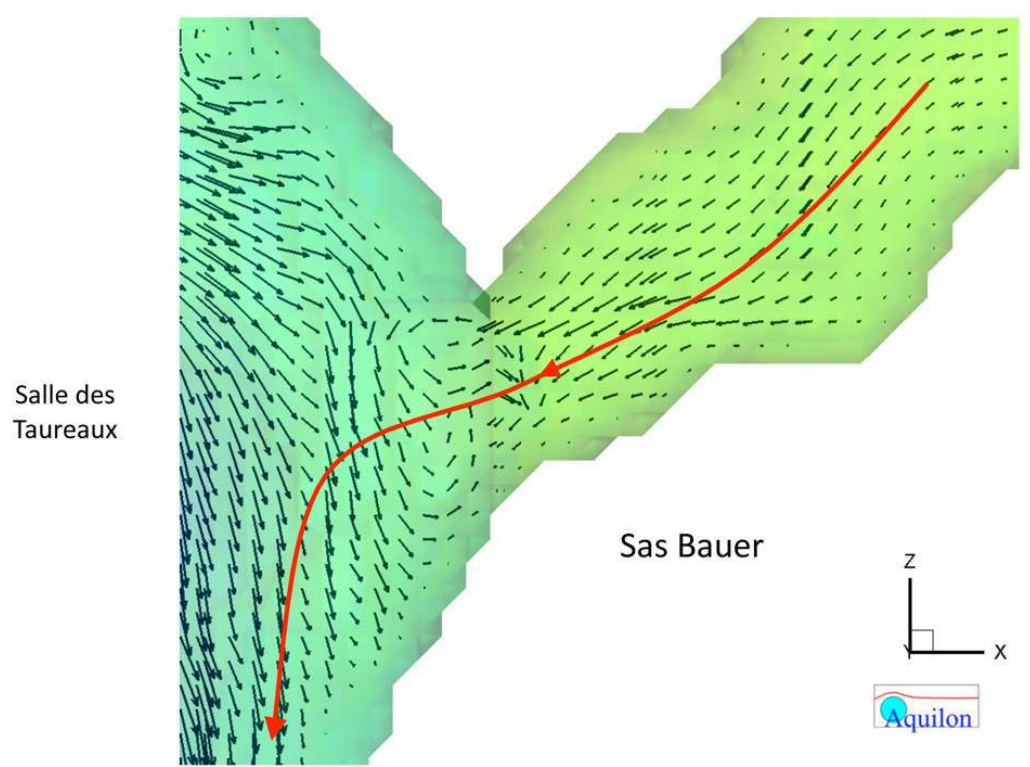

Sens des écoulements dans le Passage dans la configuration climatique de septembre 1981

Doc. D. Lacanette, P. Malaurent. @ D D. Lacanette, P. Malaurent 
Figure 20

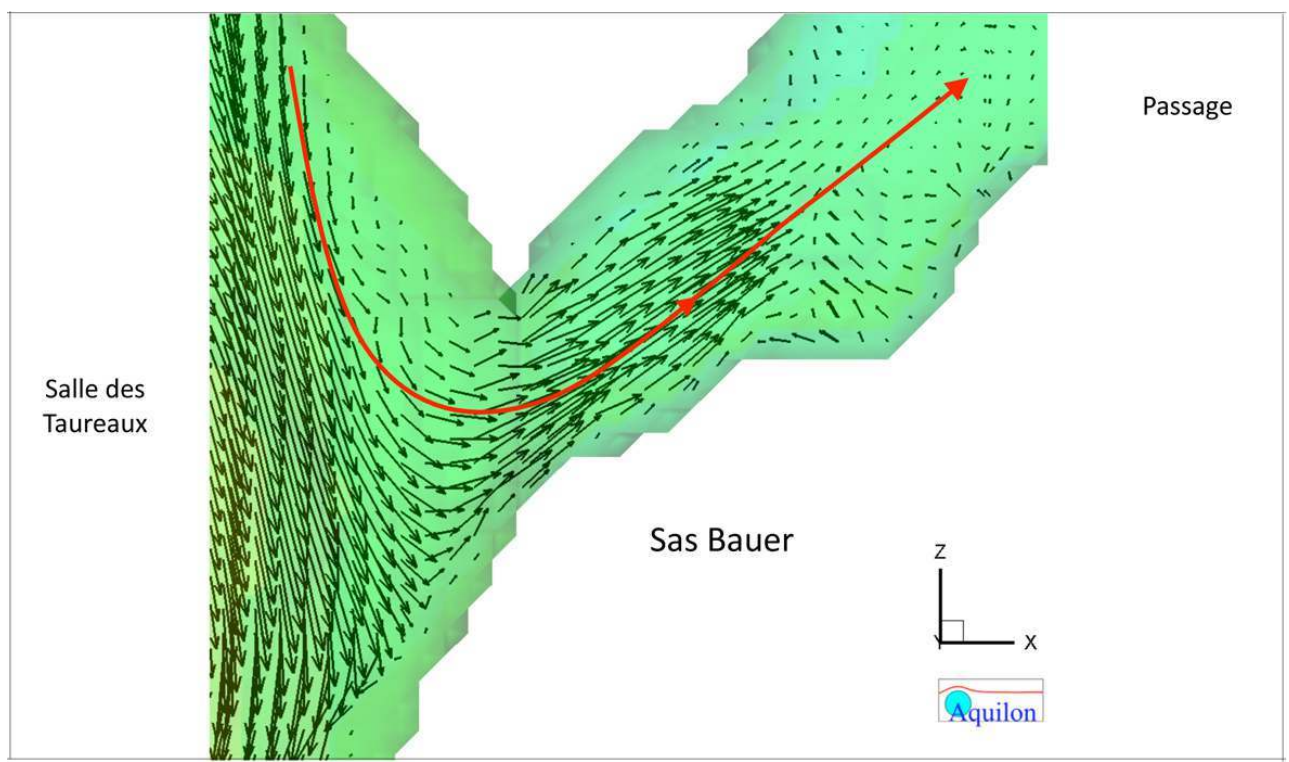

Sens des écoulements dans le Passage dans la configuration climatique de décembre 1999

Doc. D. Lacanette, P. Malaurent. @ D. Lacanette, P. Malaurent

Les figures 21,2223 et 24 soulignent la trajectoire d'une particule virtuelle lâchée au même endroit pour deux simulations correspondant à deux configurations thermiques. Dans les figures 21 et 22, la particule est lâchée au bout de la galerie du Mondmilch, et aux figures 23 et 24, elle est lâchée au fond du diverticule axial. Dans les deux cas, en septembre 1981, la particule se dirige vers l'entrée de la cavité, tandis qu'en décembre 1999, elle se dirige vers le fond de la galerie de droite. (fig. $\left.n^{\circ} 21, n^{\circ} 22, n^{\circ} 23, n^{\circ} 24\right)$ 
Figure 21

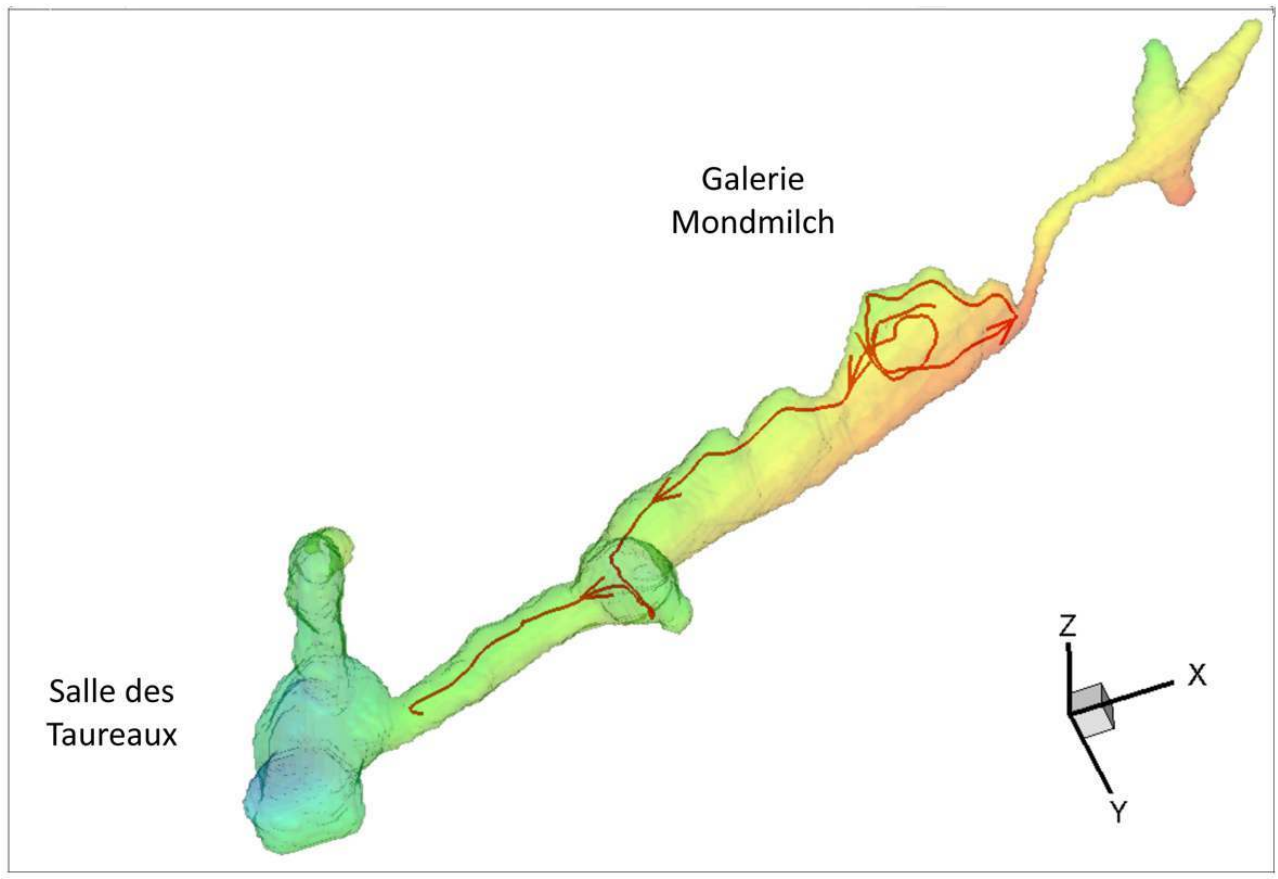

Trajectoire d'une particule virtuelle lâchée au fond de la galerie du Mondmilch dans la configuration climatique de septembre 1981

Doc. D. Lacanette, P. Malaurent. @ D. Lacanette, P. Malaurent 
Figure 22

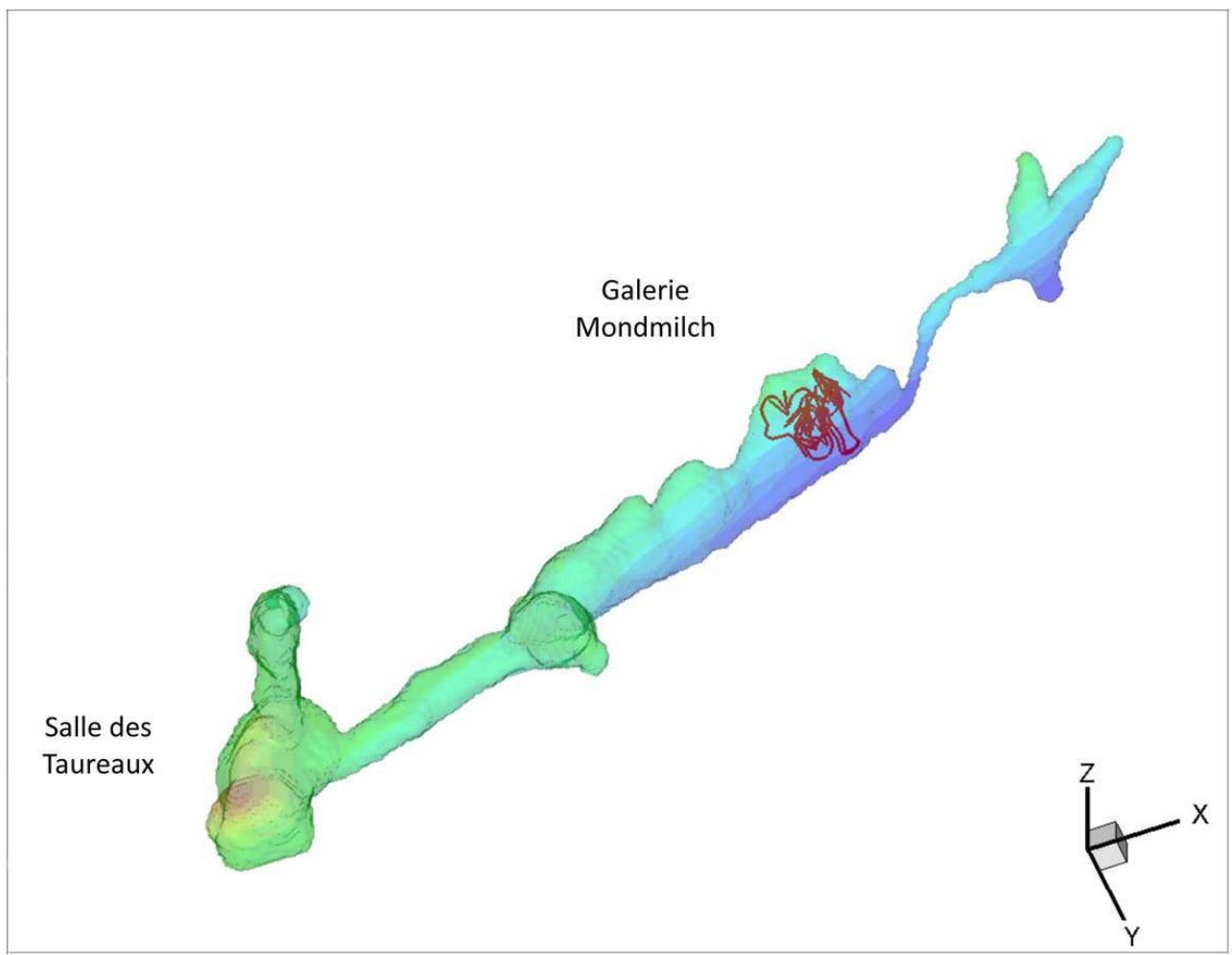

Trajectoire d'une particule virtuelle lâchée au fond de la galerie du Mondmilch dans la configuration climatique de décembre 1999

Doc. D. Lacanette, P. Malaurent. @ D D. Lacanette, P. Malaurent 
Figure 23

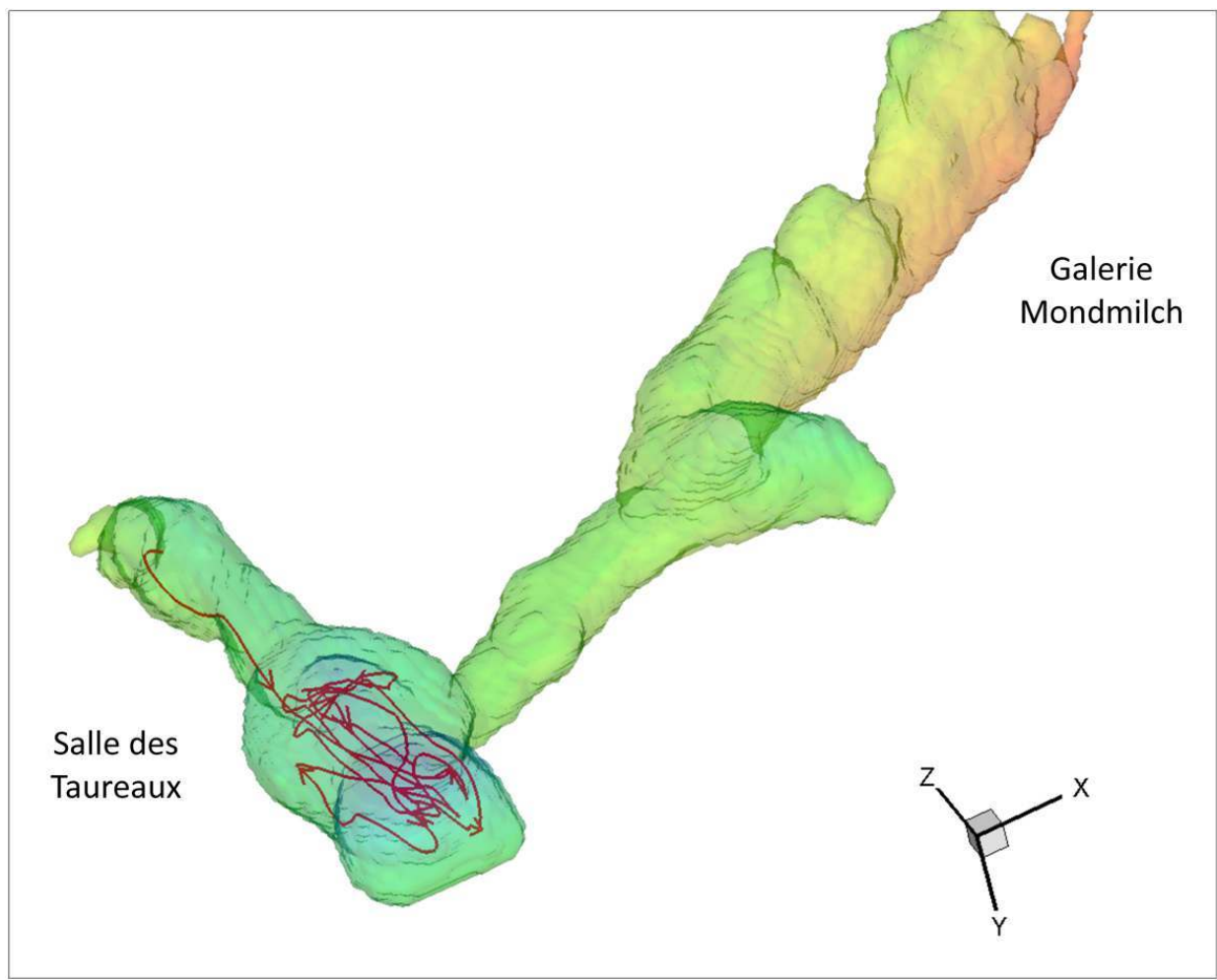

Trajectoire d'une particule virtuelle lâchée au fond du Diverticule axial dans la configuration climatique de septembre 1981

Doc. D. Lacanette, P. Malaurent. @ ( D. Lacanette, P. Malaurent 


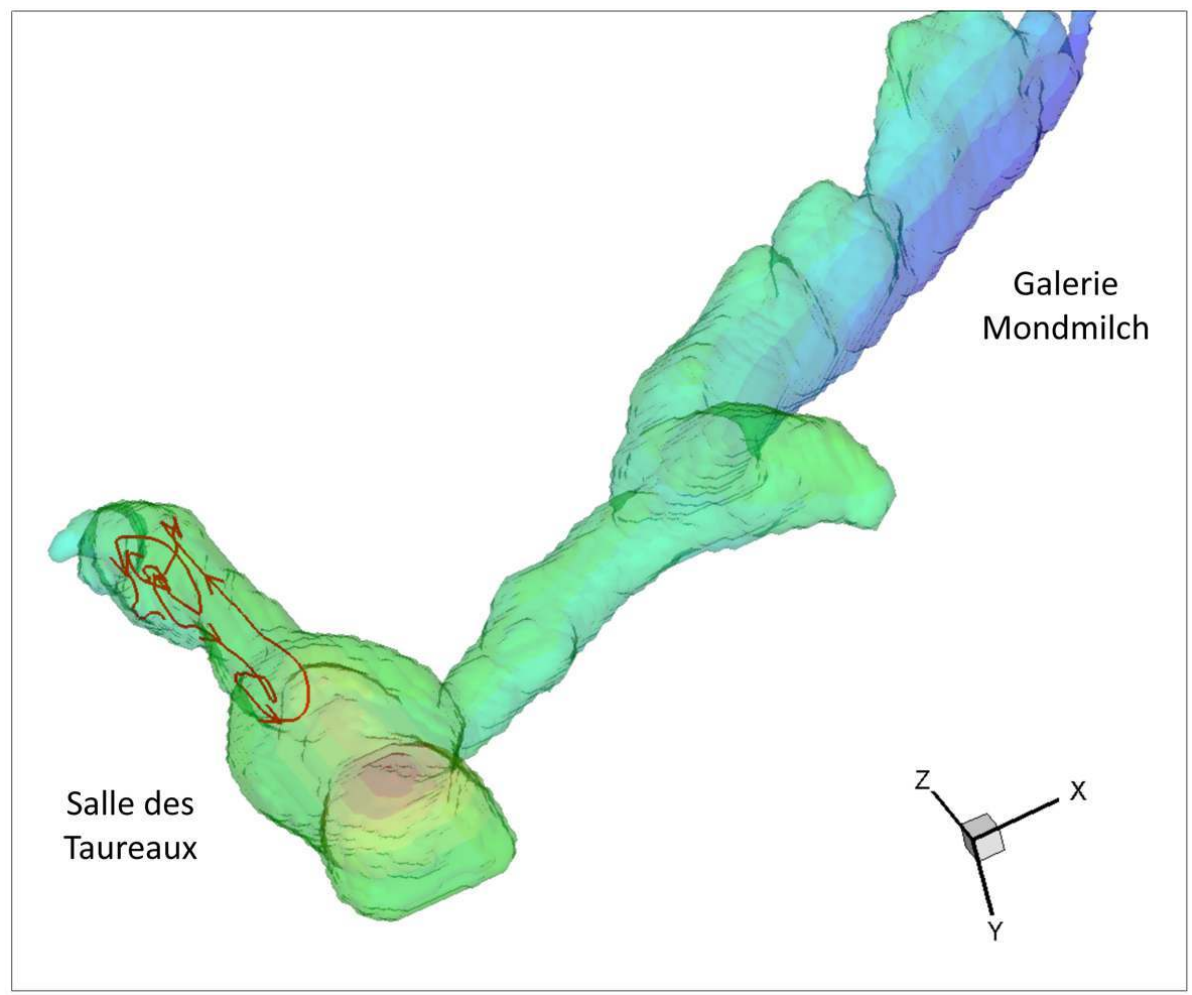

Trajectoire d'une particule virtuelle lâchée au fond du diverticule axial dans la configuration climatique de décembre 1999

Doc. D. Lacanette, P. Malaurent. @ D D. Lacanette, P. Malaurent

En ce qui concerne l'évacuation des particules virtuelles, l'écoulement dans la grotte est favorable dans la configuration thermique de 1981 et défavorable dans la configuration thermique de décembre 1999. L'analyse correspond aux observations faites à ces périodes dans la cavité ${ }^{7} 9$.

\section{Distribution de l'humidité aux parois sans les effets anthropiques}

Figure 25

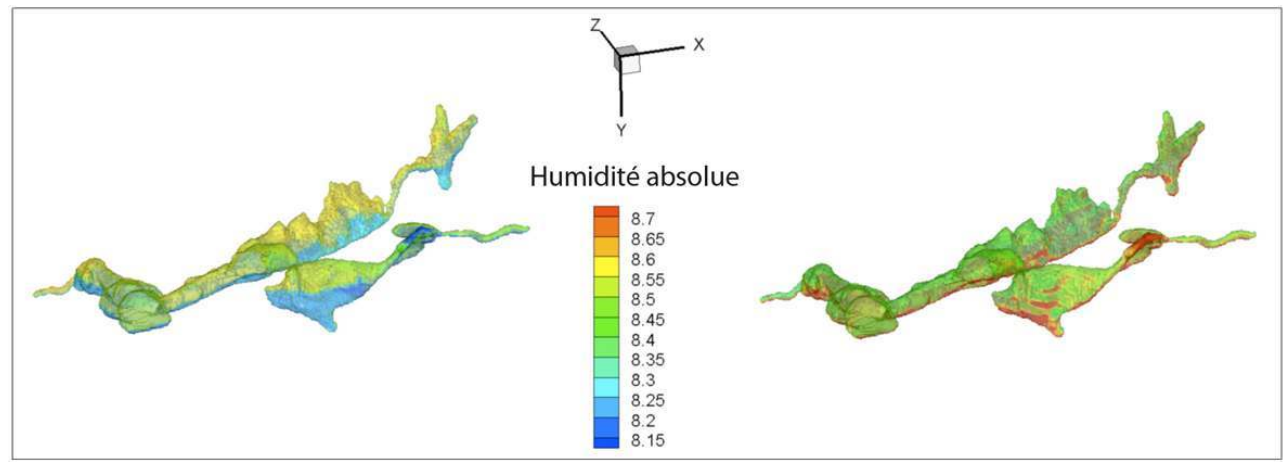

Distribution de l'humidité pour les configurations thermiques de septembre 1981 (gauche) et décembre 1999 (droite)

Doc. D. Lacanette, P. Malaurent. (c) D. Lacanette, P. Malaurent 
Le but de la simulation est de donner des informations au sujet des endroits précis des zones à fort risque de condensation. Le cas présenté ici correspond à une configuration sans effet anthropique. Il est destiné à servir de base pour des études futures, comme l'introduction du système de régulation de l'air, la présence humaine, ou encore l'introduction de points chauds et froids. La figure 25 montre la distribution du taux d'humidité absolue pour les deux configurations thermiques de septembre 1981 et décembre 1999. En ce qui concerne le taux d'humidité aux parois de la cavité, il est globalement plus élevé en décembre 1999, à cause du réchauffement de la température dans la grotte. De plus, il est concentré sur les voûtes en septembre 1981 et au sol en décembre 1999. (fig. $\mathbf{n}^{\circ}$ 25)

Figure 26

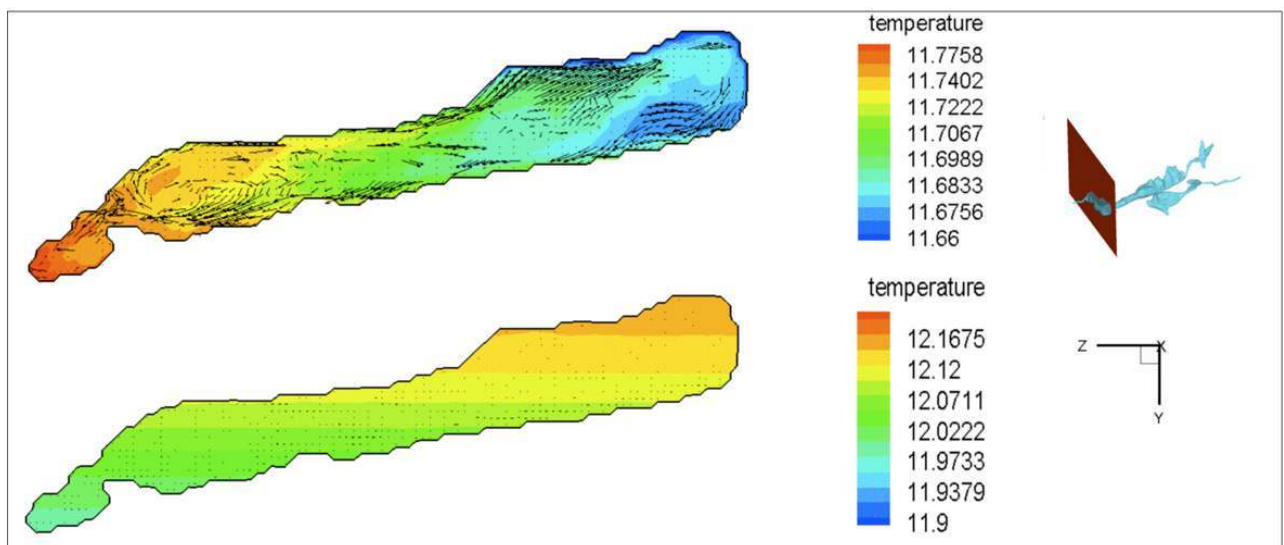

Distribution de la température pour les configurations thermiques de septembre 1981 (haut) et décembre 1999 (bas) - les vitesses sont de 0,01 m.s-1 en septembre 1981 et de 0,001 m.s-1 en décembre 1999

Doc. D. Lacanette, P. Malaurent. (c) D. Lacanette, P. Malaurent

Sur les coupes de la figure 26, on peut observer l'inversion de la température entre les deux périodes. En septembre 1981, les voûtes sont plus froides que le sol de la grotte et les courants de convection sont établis avec une vitesse moyenne de $0,01 \mathrm{~m} \cdot \mathrm{s}^{-1}$. En décembre 1999, le sol est plus froid que les voûtes, il n'y a pratiquement aucun courant de convection, l'air est stratifié et les vitesses de $0,001 \mathrm{~m} \cdot \mathrm{s}^{-1}$. La comparaison donne une information intéressante: les vitesses en décembre 1999 étaient 10 fois plus faibles que les vitesses en septembre 1981, à cause de l'inversion de température dans la colline environnante. (fig. $\mathbf{n}^{\circ} \mathbf{2 6}$ )

\section{Distribution de l'humidité aux parois avec des effets anthropiques}

La présence humaine est modélisée par l'influence thermique et hydrique de trois personnes et deux néons situés dans l'abside pendant sept heures, soit un total de vingtet-une heures de présence, de $500 \mathrm{~W}$ et de $840 \mathrm{~g}$ de vapeur. 
Figure 27

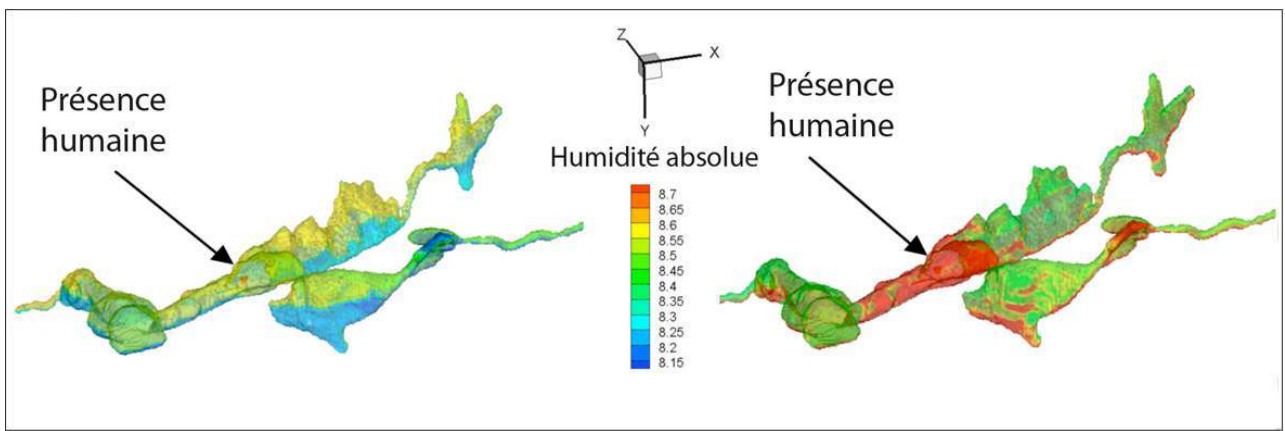

Distribution de l'humidité sur les parois de la grotte avec une présence humaine (pointée par une flèche) pour les configurations thermiques de septembre 1981 (gauche) et décembre 1999 (droite)

Doc. D. Lacanette, P. Malaurent. @ D D. Lacanette, P. Malaurent

Si l'on s'attache à regarder la répartition de l'humidité plus en détail sur des coupes, on observe à la figure 28 qu'en septembre 1981, la présence humaine accroît le taux d'humidité dans le passage et une petite partie de la nef, tandis qu'en décembre 1999, figure 29, la présence humaine augmente le taux d'humidité absolue dans une intensité plus forte et une zone plus large (jusqu'à la salle des Taureaux). En comparant les deux configurations thermiques avec la présence humaine, le taux d'humidité est beaucoup plus élevé en décembre 1999, la perturbation générée est plus grande. (fig. $\mathbf{n}^{\circ} \mathbf{2 8}, \mathbf{n}^{\circ} 29$ )

Figure 28

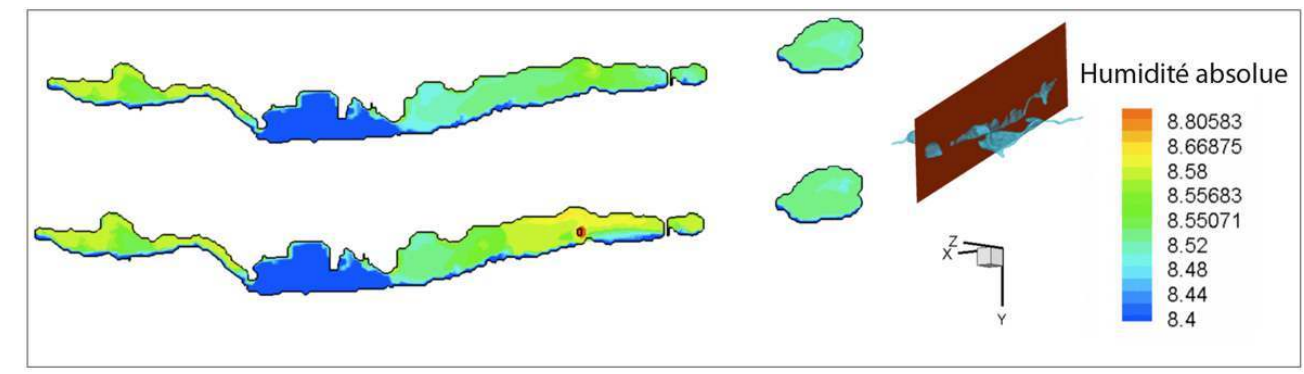

Visualisation de l'humidité absolue sur une coupe Passage / Galerie Mondmilch pour la configuration thermique de septembre 1981 sans présence humaine (haut) et avec (bas)

Doc. D. Lacanette, P. Malaurent. @ D D. Lacanette, P. Malaurent 


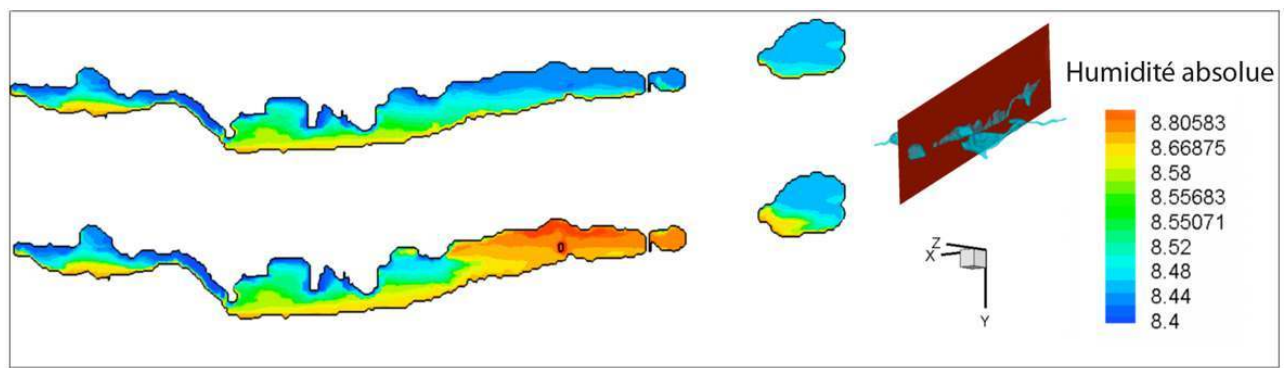

Visualisation de l'humidité absolue sur une coupe Passage / Galerie Mondmilch pour la configuration thermique de décembre 1999 sans présence humaine (haut) et avec (bas)

Doc. D. Lacanette, P. Malaurent. (c) D. Lacanette, P. Malaurent

La figure 30 présente la même configuration que la précédente avec une vue différente. Le taux d'humidité absolue est beaucoup plus élevé en décembre 1999. Les vitesses dans l'abside sont presque les mêmes dans les deux configurations et ceci est lié à la proximité de la source de la perturbation, la présence humaine. La vitesse générée par l'humain est égale à la vitesse dans la grotte en septembre 1981 sans humain et 10 fois supérieure à celle de décembre 1999 sans humain. (fig. $\mathbf{n}^{\circ}$ 30)

\section{Figure 30}

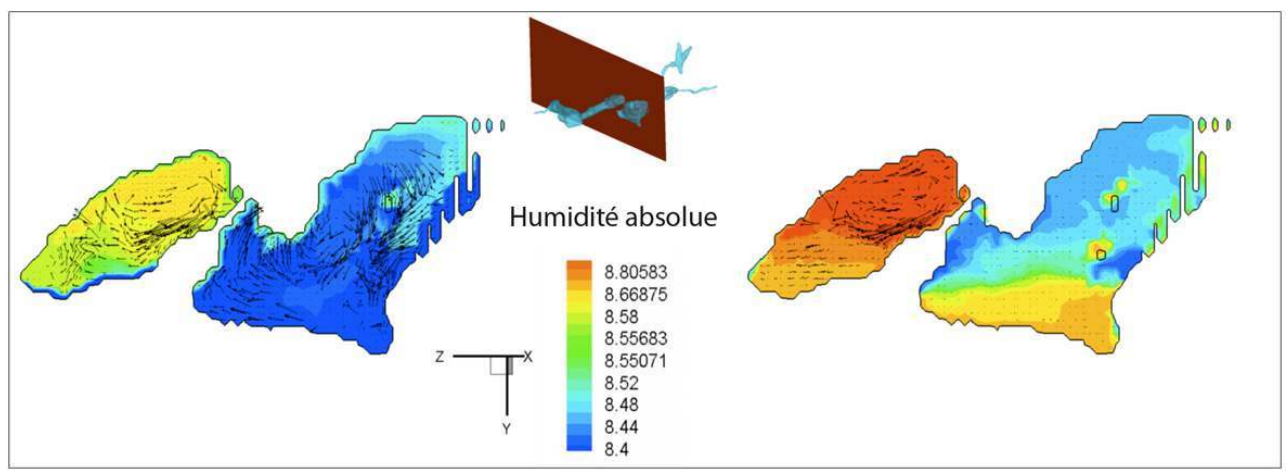

Visualisation de l'humidité absolue et des vitesses sur une coupe Abside /Puits avec présence humaine pour la configuration thermique de septembre 1981 (gauche) et décembre 1999 (décembre) Doc. D. Lacanette, P. Malaurent. @ D D. Lacanette, P. Malaurent

La figure 31 montre que la présence humaine dans le passage au niveau de l'abside n'influence pas les écoulements dans la salle des Taureaux et dans le diverticule axial en septembre 1981. Ainsi, la valeur de la vitesse générée par la perturbation, la présence humaine, est égale à la valeur des vitesses sans la perturbation, c'est-à-dire environ $0,01 \mathrm{~m} \cdot \mathrm{s}^{-1}$. (fig. $\mathbf{n}^{\circ} \mathbf{3 1}$ ) 


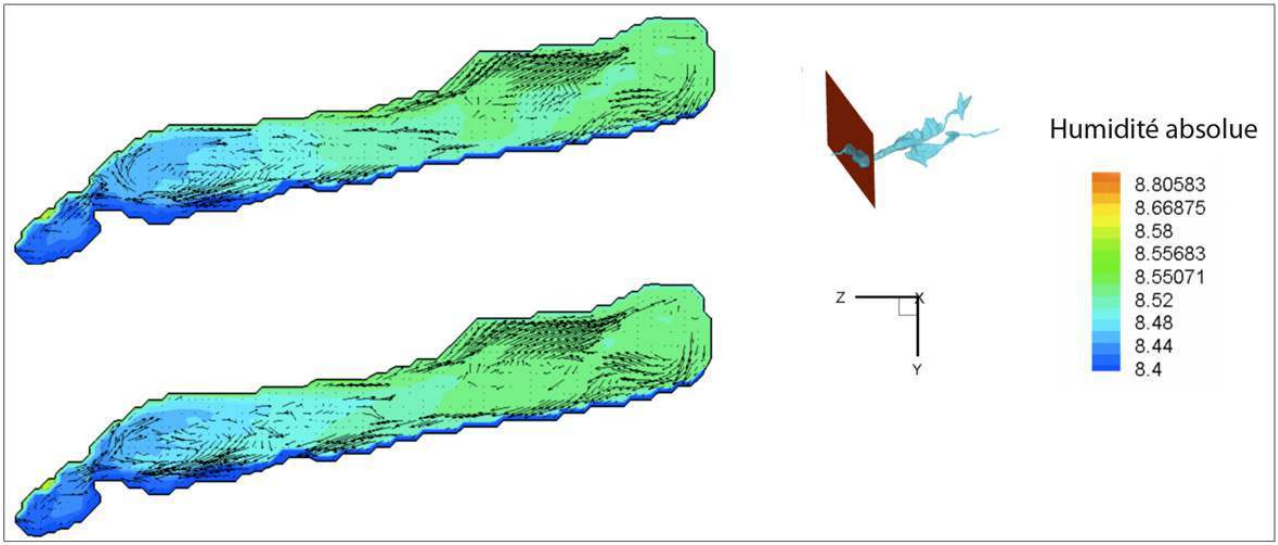

Visualisation de l'humidité absolue et des vitesses sur une coupe diverticule axial / Salle des Taureaux pour la configuration thermique de septembre 1981 sans présence humaine (haut) et avec (bas)

Doc. D. Lacanette, P. Malaurent. (c) D. Lacanette, P. Malaurent

À l'inverse, dans la figure 32, pour la configuration thermique de décembre 1999, la présence humaine a un impact sur le taux d'humidité absolue dans la salle des Taureaux. La température et le taux d'humidité sont stratifiés à cette période. L'introduction d'une source chaude augmente les vitesses et disperse le taux d'humidité dans une zone large. Ici, la valeur de la vitesse générée par la perturbation et la présence humaine est 10 fois plus élevée que la valeur des vitesses sans perturbation. (fig. $\mathbf{n}^{\circ} \mathbf{3 2}$ )

Figure 32

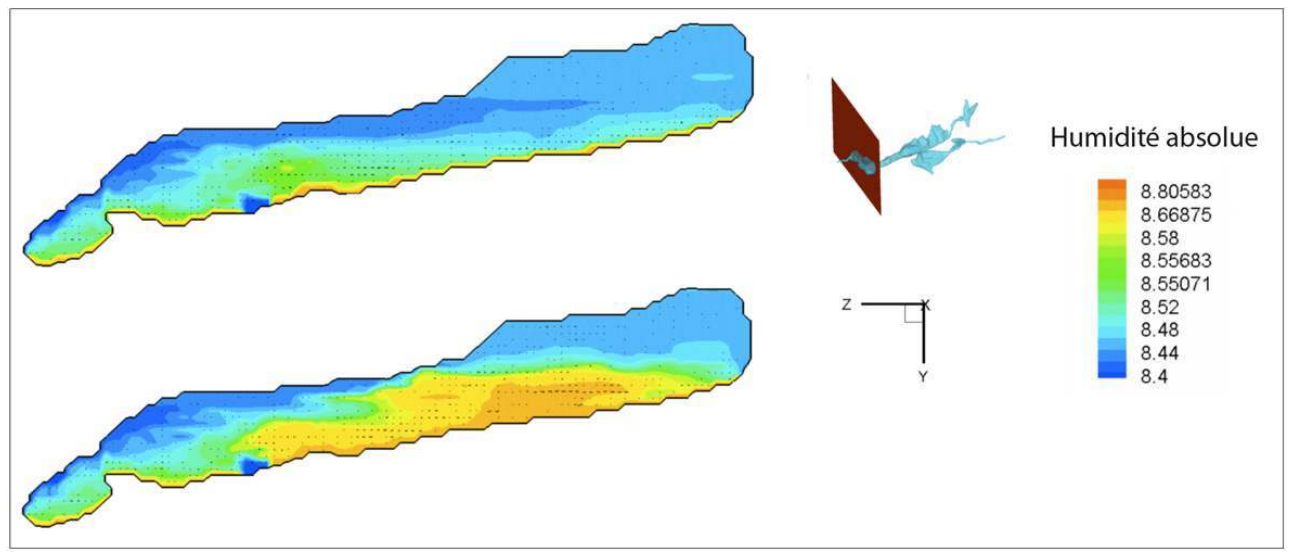

Visualisation de l'humidité absolue et des vitesses sur une coupe diverticule axial / Salle des Taureaux pour la configuration thermique de décembre 1999 sans présence humaine (haut) et avec (bas)

Doc. D. Lacanette, P. Malaurent. @ D. Lacanette, P. Malaurent

Finalement, une correspondance entre le changement climatique local et les problèmes de conservation se dégage à la figure 33. À chaque fois qu'une zone profonde est plus froide qu'une moins profonde, un problème de conservation est trouvé dans les archives. Cette inversion de température implique une stratification de l'air, comme vu précédemment, la grotte ne souffle plus et ne peut évacuer les particules potentiellement présentes. Si les stratifications étaient présentes sans les perturbations, il y aurait peutêtre moins de problèmes de conservation. (fig. $\mathbf{n}^{\circ}$ 33) 
Figure 33

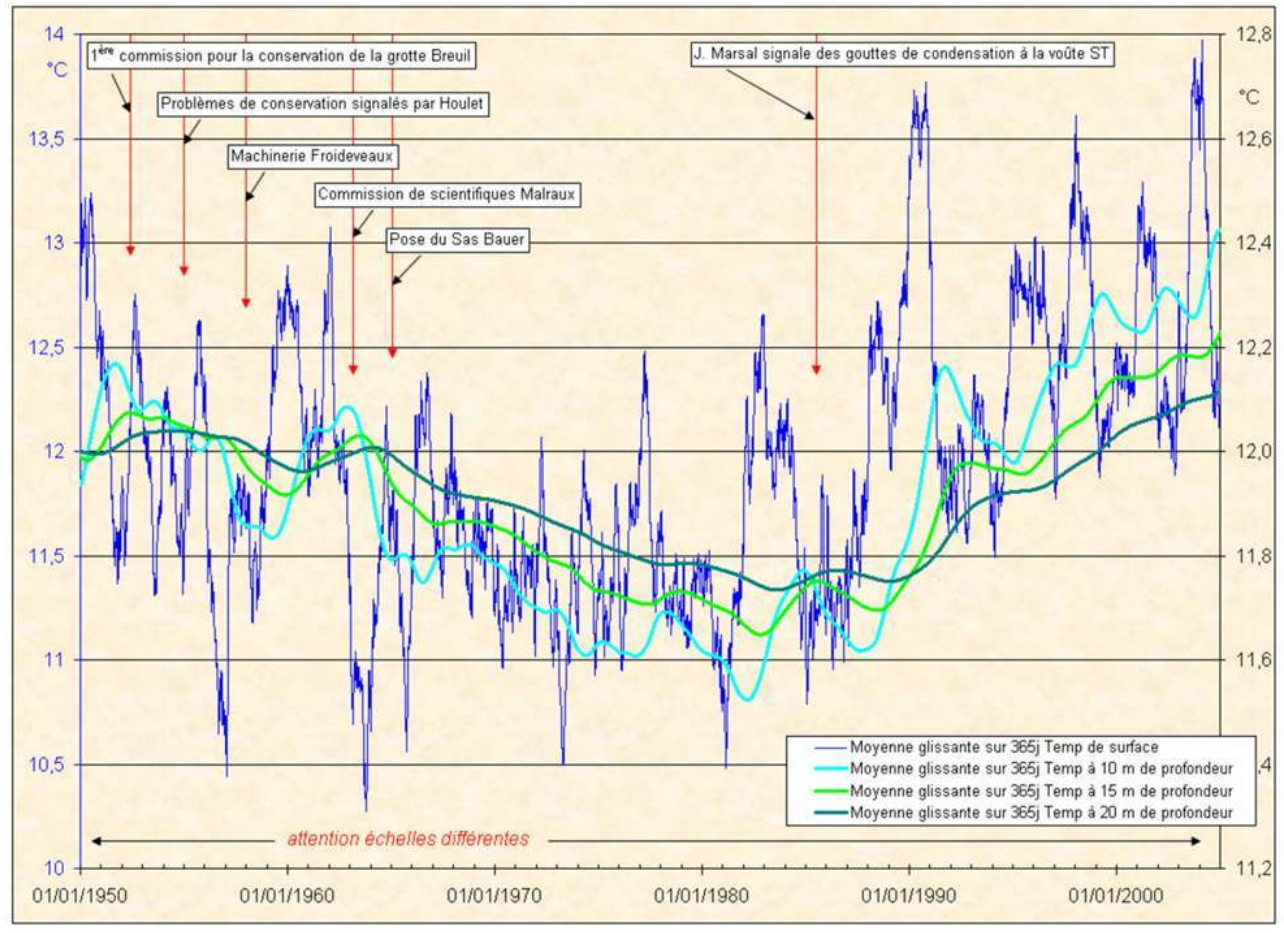

Correspondance entre les changements climatiques et les problèmes de conservation

Doc. D. Lacanette, P. Malaurent. @ D. Lacanette, P. Malaurent

C'est la présence humaine combinée à la stratification qui cause les problèmes de conservation : le taux d'humidité, le dioxyde de carbone, la pollution ... amenée par les visiteurs ne sont plus évacués vers l'extérieur dans ce cas mais doivent se disperser dans les parois.

\section{Conclusion et perspectives}

Les conditions climatiques spécifiques dans la grotte de Lascaux ont été discutées et la grande dépendance du climat extérieur avec les paramètres climatiques intérieurs a été montrée.

Le simulateur Lascaux a été introduit et détaillé, des simulations ont été réalisées pour comprendre les effets anthropiques sur la conservation de la grotte à différentes époques. Il a été montré que c'est la modification du climat qui rend la grotte plus sensible aux perturbations telles que la présence humaine.

Deux configurations climatiques ont été étudiées, représentant deux états thermiques de la cavité très différents. Il a été montré que dans la configuration thermique de septembre 1981 l'atmosphère de la grotte est relativement homogène et brassée tandis qu'en décembre 1999, elle est plus confinée.

La modification du climat local dans le milieu environnant la cavité implique une sensibilité plus forte de la grotte à la présence humaine. C'est la combinaison des inconvénients de la présence humaine avec les modifications des configurations thermiques qui causent les problèmes de conservation. 
50 En ce qui concerne les perspectives de la simulation, différents scénarios de présence humaine seront réalisés pour donner une estimation du temps de relaxation de la grotte et pour améliorer la distribution de la présence humaine à l'échelle de la semaine. De plus, la connaissance de l'impact de l'évolution du climat sur les écoulements à l'intérieur de la grotte est très importante, plusieurs scénarios d'évolution pourront être réalisés. Des travaux sur l'évolution du système d'assistance climatique sont également envisagés. (fig. $n^{\circ}$ 34)

Figure 34

\section{inp lnstitut national}

Communication présentée lors du séminaire ART RUPESTRE : LA 3D UN OUTIL DE MÉDIATION DU RÉEL INVISIBLE ? qui s'est tenu du 4 au 6 juin 2008 à Angles-sur-l'Anglin, coordonné par l'Institut national du patrimoine, avec la participation de la direction de l'architecture et du patrimoine

Les auteurs remercient le directeur régional des Affaires culturelles d'Aquitaine, le conservateur régional des Monuments historiques d'Aquitaine, le conservateur régional de l'Archéologie d'Aquitaine et le président du Comité scientifique de la grotte de Lascaux pour leur collaboration, ainsi que la direction régionale des Affaires culturelles d'Aquitaine pour son soutien financier. Les auteurs remercient également l'IDRIS et le CINES (No. TER2237-2006) pour les moyens de calcul mis à leur disposition, ainsi que le Conseil régional d'Aquitaine pour le soutien financier ayant permis d'acquérir le cluster de 256 processeurs présent au laboratoire TREFLE.

\section{NOTES}

1. Brunet, Jacques, Malaurent, Philippe, Vouvé, Jean. Lascaux, histoire d'un difficile sauvetage. Archéologia, 1997, 332, p. 24-35.

2. Sire, Marie-Anne. Des restaurateurs au chevet des peintures de Lascaux. De l'élimination des champignons au constat d'état. In Les grottes ornées. Monumental, 2006, Paris : Monum Éditions du Patrimoine, p. 68-75.

3. Orial, Geneviève, Mertz, Jean-Didier. Lascaux: une grotte vivante. Étude et suivi des phénomènes microbiologiques. In Les grottes ornées. Monumental, 2006, Paris : Monum Éditions du Patrimoine, p. 76-78.

4. Malaurent, Philippe, Lastennet, Roland, Brunet, Jacques. Une grotte sous influence: l'environnement hydrogéologique et climatique de la grotte de Lascaux. In Les grottes ornées. Monumental, 2006, Paris : Monum Éditions du Patrimoine, p. 88-93.

5. Lacanette, Delphine, Malaurent, Philippe, Caltagirone, Jean-Paul, VIncent, Stéphane. A model of thermal and aeraulic flows in the cave of Lascaux. International Association for Mathematical Geology, XI $^{\text {th }}$ International Congress, 3-8 September 2006, Liège, Belgium.

6. Lacanette, Delphine, Caltagirone, Jean-Paul. Le simulateur Lascaux: un outil d'aide à la décision pour l'avenir de la préhistoire. In Les grottes ornées. Monumental, 2006, Paris : Monum Éditions du Patrimoine, p. 94-97. 
7. Caltagirone, Jean-Paul, Glockner, Stéphane, Vincent, Stéphane. Aquilon : outil de modélisation en mécanique des fluides et transferts. Voir le site: http://www.trefle.ubordeaux1.fr/aquilon.

8. Lacanette, Delphine, Malaurent, Philippe, Caltagirone, Jean-Paul, Brunet, Jacques. Étude des transferts de masse et de chaleur dans la grotte de Lascaux : le suivi climatique et le simulateur, Karstologia, 50, 2007, p. 19-30.

9. Malaurent, Philippe, Brunet, Jacques, Lacanette, Delphine, Caltagirone, Jean-Paul. Contribution of numerical modelling of environmental parameters to the conservation of prehistoric cave paintings: the example of Lascaux Cave, Conservation and management of archaeological sites, 8, 2006, p. 1-11.

\section{RÉSUMÉS}

La simulation numérique en mécanique des fluides est appliquée ici à la conservation du patrimoine et plus particulièrement à la grotte de Lascaux en Dordogne. Un relevé laser 3D très précis de la grotte de plus de 150 millions de points a servi de base au simulateur Lascaux, outil numérique permettant de tester en laboratoire, sur ordinateur, les modifications à apporter éventuellement à la cavité et de vérifier la façon dont elle réagit avant des réaliser ces changements in situ. Il est en cela un outil de conservation préventive innovant.

Numerical simulation in fluid mechanics is applied here to the conservation of the patrimony and more particularly to the Lascaux cave, in Dordogne, France. A very accurate 3D laser survey of the cavity of more than 150 million points was used as a base of the Lascaux simulator, a numerical tool allowing to test in laboratory, on a computer, the modifications to bring eventually to the cave, and to check the way it reacts before achieving these modifications in situ. The simulator is an innovative conserving tool for preventive conservation.

\section{INDEX}

Mots-clés : maillage, diverticule axial, salle des Taureaux, comité scientifique, corrosion, microorganismes, hygrométrie, calcite, mousses, algues vertes, patrimoine mondial de l'Unesco, relevé laser 3D, dordogne, grotte de Lascaux, conservation du patrimoine, mécanique des fluides, simulation numérique, simulateur Lascaux, grottes ornées

\section{AUTEURS}

\section{DELPHINE LACANETTE}

Chercheur contractuel, Laboratoire Transferts, Écoulements, Fluides, Énergétique (TREFLE), UMR CNRS 8508 Site ENSCPB. lacanette@enscpb.fr 


\section{PHILIPPE MALAURENT}

Ingénieur d'études, Laboratoire Géosciences, Hydrosciences, Matériaux, Construction (GHYMAC). philippe.malaurent@u-bordeaux1.fr 